\title{
Combien d'eau utilise-t-on et use-t-on ? Pour quoi faire ?*
}

\author{
How much water do we use? For what uses? \\ par Jean Margat, \\ BRGM
}

Water uses are diversified but the use of this special "raw material" is not really taken into account in the economical statistics. The water quantities (3000 to 4000 billions cubic meters globally) vary between the countries and the uses : it depends upon the climate (if it is or not necessary to irrigate) and the level of development. In the world, the first use is irrigation (70\%), followed by industry and thermal power plants cooling $(22 \%)$ and drinking water for people $(8 \%)$. But these percentages vary from one country to another.

After a global increasing of the used quantities along the XXth century (multiplied by 6), we see important contrasts between the developed countries (low growth) and the others (high growth with enlarged irrigation). The ratio between the used quantities and the natural renewing resources, globally of $8 \%$, varies from 1 to over $100 \%$ in some countries that exploit their water reserve).

Moreover, the water used quantities generate waste water production (those of cities and industries have grown 20 times along the XXth century).

\section{I $\square$ INTRODUCION}

Faut-il d'abord rappeler quels services l'humanité demande à l'eau ? Quelles sont la nature et les finalités des utilisations de l'eau ? (encadré 1). Nourrir, laver, travailler, chauffer ou refroidir, éteindre, climatiser, former un milieu de vie, une voie de transport ou une ligne de défense, un espace de jeu ou un élément de paysage : aucun élément naturel n'a autant d'usages, pour la plupart sans substitut. Mais ces usages ne font pas appel aux mêmes fonctions de l'eau et mettent en jeu des quantités aussi variées que les qualités. Pour chaque individu, comme pour les édiles, l'eau est d'abord un bien de consommation domestique, boisson et agent culinaire, facteur d'hygiène, de confort, de décor et de loisirs... Mais les utilisations d'eau comme facteur de production agricole et piscicole, industrielle et énergétique, mobilisent des volumes largement supérieurs et de qualités voulues bien différentes.

Peut-on pour autant parler de besoins en eau ? Entre désirs et nécessités, le concept théorique et normatif de besoin sert plutôt de référence pour apprécier si les demandes réelles actuelles sont insuffisantes (restrictions) ou excessives (gaspillage), ou encore d'indicateur pour projeter des demandes futures. On sait combien ces besoins diffèrent suivant les usages et leur efficacité voulue, en volume comme en qualité requise, aussi bien qu'en proportion

* Cet article est extrait du livre "L'eau en questions" paru aux éditions Romillat. consommée nette et en usure de qualité par les charges de matières des rejets. Même pour chaque usage pris à part, une grande variété de demandes et de "consommations" unitaires en quantité - par usager, par unité de produit, par hectare irrigué... - se rencontre dans le monde et dans chaque pays, suivant les comportements des usagers, les conditions climatiques, les procédés industriels ou les pratiques d'arrosage et aussi suivant les coûts de production ou de fournitures de l'eau. Aussi des standards moyens en cette matière traduisent-ils mal des réalités diversifiées (encadré 2).

De plus, ces besoins ont évolué et peuvent encore changer : les besoins sociaux de "consommation" en eau suivant le niveau de vie, les besoins en eau facteur de production suivant les techniques. Les hygiénistes de la fin du XVIII ${ }^{e}$ siècle évaluaient les besoins domestiques à 20 ou 30 litres journaliers par personne ; c'est encore la "consommation" actuelle dans beaucoup de villes d'Afrique, tandis qu'en Europe elle a dépassé 100 litres dans la plupart des villes dès les années 50, atteignant souvent 200 litres ou plus actuellement, et qu'elle approche 400 à 500 litres, dans plusieurs agglomérations nord-américaines. En sens inverse, beaucoup de besoins unitaires d'eau à usages industriels ont décru, parfois fortement, de même que ceux de l'agriculture irriguée moderne. Nonobstant ces relativités et ces évolutions, des besoins en eau intrinsèques peuvent-ils être définis pour diagnostiquer des pénuries d'eau présentes ou futures et justifier des efforts pour les réduire (encadré 3) ? Cette question se relie à la distinction entre besoins et demandes (encadré 4). 


\section{1. -PRÉLEVER, PRODUIRE, UTILISER, CONSOMMER, USER}

Pour parler le même langage :

- Prélever, c'est prendre de l'eau dans le milieu naturel (exploiter, capter) soit pour s'approvisionner directement, soit pour produire l'eau à distribuer aux usagers desservis, soit encore pour mettre "hors d'eau" le sol ou le sous-sol (exhaure des mines...) ; dans ce dernier cas, on ne prélève pas l'eau pour l'utiliser mais pour utiliser l'espace qu'elle occupe.

- Produire de l'eau c'est la rendre disponible et apte à tel ou tel usage à partir d'une matière première exploitée, soit, le plus souvent, de l'eau douce prélevée dans le milieu naturel (ressource), soit aussi de l'eau saumâtre ou de l'eau de mer (à dessaler) ou encore de l'eau usée (à régénérer). Les opérations de production comprennent la chaîne plus ou moins complexe des travaux d'aménagement et de maîtrise de l'eau dans le milieu, de prélèvements, de traitement (par exemple de potabilisation) et de stockage, ou de "fabrication" et de conditionnement par différents procédés industriels (dessalement...), en amont de la distribution ou d'usage direct par l'exploitant auto-consommateur.

- Utiliser l'eau, c'est s'en servir à diverses fins, en en faisant différents usages, soit comme bien de consommation (services domestiques, alimentation), soit comme facteur de production. Les utilisations de l'eau se définissent par rapport aux objectifs sociaux et économiques ; elles se classent en secteurs d'utilisation : domestique, municipal, agricole, industriel,... Les usages se définissent par les fonctions de l'eau qu'ils appliquent : abreuver, nourrir, laver, chasser, dissoudre, refroidir ou chauffer, faire flotter, pousser, etc. L'eau peut aussi être utilisée dans le milieu naturel (rivières, lacs...) sans être prélevée (utilisations in situ : production d'énergie hydraulique, navigation, pêche, loisirs...).

- Consommer équivaut pour l'usager "consommateur", plus particulièrement lorsqu'il est desservi par un distributeur, à acquérir l'eau distribuée, à "prélever sur le réseau" (comme il "consomme l'électricité"). Pour le distributeur, la "consommation" est, en sens inverse, la quantité d'eau qu'il délivre, comptabilise et facture. Par rapport au milieu naturel, reproducteur de la ressource, consommer c'est plutôt ne pas retourner après usages une partie des quantités d'eau qui ont été prélevées et utilisées : cette consommation nette ou finale correspond aux quantités d'eau évaporée ou transpirée au cours des usages (ou encore rejetée après usages dans un autre milieu, comme la mer).

- User l'eau, c'est réduire, en en faisant usage, ses aptitudes à servir, tout spécialement ses qualités : la charger de déchets, de substances dissoutes ou de calories (quand elle sert à refroidir) ; ou encore diminuer son potentiel d'énergie hydraulique en la faisant chuter. Tout usage use l'eau et l'eau usée ne peut resservir aux mêmes usages qu'après régénération de son potentiel (pureté, etc.) soit par la Nature - dans les limites où elle le peut -, soit par des traitements d'épuration. Retourner de l'eau usée au milieu naturel dans un état dégradé (par rapport à son état originel), donc même en partie épurée, revient à consommer la ressource en qualité et à exploiter la capacité d'autoépuration du milieu.

\section{Q QUELLE QUANTITÉ D'EAU L'HUMANITÉ UTILISE-T-ELLE EN GROS DANS LE MONDE ENTIER ?}

Les essais de chiffrage ne sont pas très convergents : les estimations varient suivant les sources entre 3000 et plus de 4000 milliards de $\mathrm{m}^{3}$ annuels, mais ne procèdent pas d'une même approche (les statistiques et les calculs à partir de facteurs présumés des demandes y prennent des parts variables) et ne paraissent pas reposer sur des bases cohérentes, ce qui explique les écarts.

Deux estimations globales modernes peuvent être citées : voir tableau 1.

\begin{tabular}{|l|c|c|c|}
\hline Référence & J. Margat' & \multicolumn{2}{|c|}{ I. Shiklomanov (1996) } \\
\hline date de valeur & Fin de la décennie 80 & 1990 & 1995 \\
\hline $\begin{array}{l}\text { Prélèvements } \\
\text { (sans l'évaporation } \\
\text { des réservoirs) }\end{array}$ & 3270 & 3416 & 3572 \\
\hline $\begin{array}{l}\text { Consommation } \\
\text { finales en km/an } \\
\text { (dont : évaporation } \\
\text { des réservoirs) }\end{array}$ & 1800 & 2196 & 2285 \\
\hline
\end{tabular}

Tableau 1. Estimations des quantités d'eau utilisées dans le monde.

\footnotetext{
'Estimation en priorité par sommation de statistiques nationales inventoriées et, seulement à défaut de celles-ci, par calculs basés sur des variables exogènes facteurs des demandes

${ }^{2}$ non comprise
}

\subsection{Sait-on mesurer les utilisations présentes ?}

La connaissance des utilisations d'eau actuelles n'est pas forcément meilleure que celle des ressources. Comme celleci, elle est pour le moins inégale, selon les secteurs d'utilisation et les pays. Dans bien des cas, faute de recensement contemporain et de statistiques assez fiables et complètes, on est réduit à "prévoir le présent" par des calculs basés sur des standards moyens vraisemblables de demande en eau - par habitant, par unité de surface irriguée... - avec le risque d'estimation par excès si on les assimile à des "besoins" normés en quantité.

Les statistiques disponibles portent généralement seulement sur ce qu'on sait le moins mal compter : les volumes prélevés (ou produits). Encore sont-elles frappées d'approximations variées qui rendent les sommations et les comparaisons difficiles, entre secteurs comme entre pays ${ }^{3}$. En outre, du fait des multiples "dimensions" des demandes en eau, globaliser ces seuls volumes d'eau indifférenciés simplifie à outrance l'analyse.

Les demandes en eau en qualité sont presque uniquement déduites de la répartition des quantités par secteur d'utilisation, et elles sont aussi basées sur des normes d'usages plus que sur des constats directs. La connaissance des rejets, très indirecte, est encore plus floue. La nature et les quantités des charges de matière que les rejets d'eau usée ont pour fonc-

\footnotetext{
${ }^{3}$ Les données quantitatives par pays utilisées ici, agrégées ou traduites en cartographie, proviennent de sources nationales contemporaines ou de compilations internationales, notamment : ENGELMAN \& AI. 1993, FAO 1995, 1997. MARGat 1990, 1994, 1996, ShikLomanov 1996, OCDE 1997, UN/ECE 1993. WRI 1996.
} 
tion d'évacuer, sont sujettes à de nombreuses analyses de cas locaux, mais peu comptabilisées à l'échelle globale d'un bassin ou d'un pays.

Quant aux impacts finaux sur les qualités des eaux dans le milieu, ils ne peuvent par nature donner lieu à des statistiques globales. Rapporter tout le volume d'eau usée ou toute la masse de matière émise à tout le débit des cours d'eau d'un bassin ne fait pas une moyenne significative...

A la différence des consommations en volume les consommations en qualité ne s'additionnent pas. Les pollutions sont objets de géographie qualitative bien plus que de quantifications ; elles ne sont pas directement proportionnées aux "faits polluants" (aux émissions de matières), mais dépendent tout autant des sensibilités des milieux récepteurs et des objectifs de qualité attribués à ceux-ci. L'utilisation des ressources se mesure mieux que leur usure.

\subsection{Les demandes en eau en quantité sont très diversifiées et inégales dans le monde}

Comme les ressources naturelles, les utilisations d'eau ont leur géographie. A l'échelle macroscopique des pays ellemême, la diversité et la multiplicité des facteurs et des motifs de demandes en eau - ou plus largement de sollicitations des eaux du milieu naturel - les rendent presque aussi variées et inégalement réparties dans le monde que les ressources.

Trois facteurs principaux déterminent cette variété et cette inégalité :

- D'abord, le climat qui nécessite ou non l'irrigation des cultures suivant les régions. Dans une grande partie du monde à "déficit hydrique" - zones à climat aride, semi-aride ou méditerranéen - la production agricole est subordonnée à l'irrigation, en principal ou en complément. La demande en eau d'irrigation l'emporte, souvent de beaucoup, sur toutes les autres et amplifie considérablement la demande en eau globale. L'aridité du climat grossit aussi beaucoup la consommation d'eau des retenues, par évaporation. Ainsi, par rapport aux pays à climat humide, les pays des zones arides ou semiarides sont doublement pénalisés : par la rareté des ressources et par l'amplification des besoins humains.

- Puis la répartition des populations et la densité démographique de chaque pays, même si les demandes effectives par habitant varient largement.

- Enfin, la forme et le degré de développement socio-économique, qui influencent fortement les demandes des collectivités et des secteurs de production industrielle ou énergétique, ainsi que la création des moyens de les satisfaire. Un haut degré de développement induit en général des demandes en eau effectives - et non pas seulement des besoins théoriques - plus fortes, sauf en agriculture (là où l'irrigation est nécessaire ou avantageuse) dans la mesure où les modes d'irrigation plus efficients peuvent atténuer les demandes. Toutefois les interférences des autres facteurs brouillent la relation entre les indicateurs du développement et les demandes en eau globales.

Les structures de chacun des grands secteurs d'utilisation entraînent des variétés propres de leurs demandes en eau, qui se superposent aux effets généraux de ces facteurs :

- la composition des cultures irriguées, notamment le poids relatif des plus consommatrices (riziculture) ;

- les parts respectives des différents procédés d'irrigation (gravitaire, aspersion, micro-irrigation) dont les efficiences sont très inégales ;

\section{2. — VARIÉTÉ DES "BESOINS EN EAU" UNITAIRES EN QUANTITÉ}

- "Consommation" domestique urbaine (eau potable) :

50 à 500 litres/jour/hab.

Moyenne française (Paris excepté) : $\quad 163$ l/jour en 1990

Paris (toutes consommations) : $\quad 324 \mathrm{l} / \mathrm{jour}$ en 1990,

280 en 1996

Agglomérations des pays développés : 0,5 à $4 \mathrm{~km} / 3 / \mathrm{an} / \mathrm{km}^{2}$

- "Consommation" rurale : Moyenne française/consomma-

tion domestique : $149 \mathrm{l} /$ jour/hab.

- avec desserte, en pays développés : 100 à

$200 \mathrm{l} /$ jour/hab.

- sans desserte, dans le tiers-monde : 10 à 25 l/jour

- Élevage : Gros bétail : 50 à $200 \mathrm{l} /$ jour par tête Petit bétail : 10 à $40 \mathrm{l} /$ jour par tête

- Irrigation : 2000 à $20000 \mathrm{~m}^{3} /$ an par hectare suivant le climat, les cultures et le nombre de récoltes annuelles

Pays méditerranéens : 8000 à $15000 \mathrm{~m}^{3} /$ an par hectare Égypte : $\quad 15000$ à 18000

Iraq : $\quad 22000$

Coton: $\quad 8000$ à 12000

Rizières (Asie du Sud-Est) : 15000 à 40000

Canne à sucre : $\quad 20000$ à 40000

- Production de biomasse en condition de sécheresse : $1000 \mathrm{~m}^{3}$ par tonne (consommation nette)

- Refroidissement d'une centrale thermo-électrique : en circuit ouvert : 145 à 165 litres par kWh produit en circuit fermé : 3 à 20

- Évaporation d'une retenue : 5000 à $25000 \mathrm{~m}^{3} / \mathrm{an}$ par hectare de plan d'eau suivant le climat et l'altitude

- Quelques standards de besoins industriels (suivant les procédés) :

$\begin{array}{ll}\text { Acier } & 6 \text { à } 300 \mathrm{~m}^{3} \text { par tonne de produit } \\ \text { Papier } & 80 \text { à } 1000 \\ \text { Essence (raffinage) } & 0,1 \text { à } 40 \\ \text { Bière } & 8 \text { à } 25 \\ \text { Sucre } & 3 \text { à } 400 \\ \text { Savon } & 1 \text { à } 35\end{array}$

- le taux d'urbanisation des collectivités humaines ; - les parts respectives des différentes branches industrielles, très inégalement utilisatrices d'eau : l'industrie chimique, la fabrication de pâte à papier, le raffinage du pétrole et la métallurgie sont les branches qui utilisent le plus d'eau.

En France, par exemple, 4 branches ont prélevé, en 1986, $62,3 \%$ du volume total d'eau à usages industriels $(4,84$ milliards de $\mathrm{m}^{3}$ ) :

- Chimie de base et production de fibres ou fils synthétiques : $34,3 \%$

- Industries du papier et du carton : $12,6 \%$

- Métallurgie : 7,7 \%

- Parachimie et industrie pharmaceutique : 7,7 \% (Enquête du ministère de l'Environnement publiée en 1989).

- enfin, les proportions respectives des productions d'électricité thermique (y compris nucléaire) dues à des centrales intérieures ou littorales, et, pour les premières, à système de refroidissement en circuit ouvert ou fermé : suivant ces parts, les demandes d'eau douce de refroidissement sont sans commune mesure. 


\section{3. -DES BESOINS EN EAU MINIMAUX UNIVERSELS PAR HABITANT SONT-ILS DÉFINISSABLES ?}

Pour identifier et apprécier des manques d'eau présents ou prévoir et situer d'éventuelles pénuries futures, il faut pouvoir se baser sur un concept de besoins en eau intrinsèques par habitant, définissables indépendamment des offres, à la différence des demandes en eau, pour les comparer aux ressources.

Un minimum vital et social de quantité d'eau potable nécessaire par personne du point de vue nutritionnel et sanitaire, comme facteur de bien-être, en somme de "besoins domestiques", est concevable. Encore ne peut-il être indépendant du climat et des traditions culturelles, ni d'une référence à un niveau de vie minimal... M. Falkenmark, par exemple, a estimé ce minimum ("basic household") à 100 l/jour en zone aride pour un niveau de vie moyen. Ces besoins élémentaires ne peuvent cependant être dissociés de ceux liés aux productions alimentaires, industrielles et énergétiques qui conditionnent le niveau de vie et sont estimés, toujours par M. Falkenmark, à " 5 à 20 fois" plus, soit à 180 à $730 \mathrm{~m}^{3} / \mathrm{an}$ par tête. D'où l'idée qu'un état de "pénurie absolue" s'instaurerait dès que les ressources en eau naturelles (donc incomplètement exploitables) seraient inférieures à $500 \mathrm{~m}^{3}$ /an par tête et que la situation serait tendue ("water stress") au dessous du seuil de $1000 \mathrm{~m}^{3} / \mathrm{an}$ - ou, en d'autres termes, lorsque les populations par "unité de ressource" d'un million de $\mathrm{m}^{3} / \mathrm{an}$ en moyenne dépasseraient respectivement 2000 ou 1000 habitants. Ce qui revient à définir un "minimum vital" de ressources en zone aride ou semi-aride.

Néanmoins le concept d'un standard universel de besoins en eau minimaux pour toutes utilisations confondues, même accordé aux conditions physiques les plus amplificatrices (aridité), paraît trop simplificateur car il suppose des structures d'utilisation uniformes. Quel que soit le niveau de développement économique, suivant que l'irrigation pèse un peu, beaucoup ou pas du tout dans les utilisations d'eau, ou suivant que la thermoélectricité prend une part dominante ou négligeable dans la production énergétique, à l'évidence les besoins en eau minimaux par habitant ne seront pas du tout les mêmes.

L'appréciation des "déficits" en eau dans les pays en développement, où ils sont souvent considérés comme des handicaps dans les discours médiatiques, devrait en tenir compte davantage et ne pas se référer à un seuil unique indépendant des climats.
Ainsi, les pays les plus voraces en eau dans le monde sont naturellement les plus peuplés, mais parmi eux s'entremêlent des pays très développés, aussi bien que des pays "en développement" et irriguant massivement (Fig. 1). Les 29 pays qui utilisent chacun plus de $20 \mathrm{~km}^{3}$ d'eau par an rassemblent $69 \%$ de la population mondiale (1990) et prélèvent ensemble près de $3000 \mathrm{~km}^{3} / \mathrm{an}$, soit $86 \%$ de toute l'eau utilisée dans le monde actuellement. Parmi eux, cinq pays, les plus utilisateurs d'eau du monde, prélèvent chacun plus de $100 \mathrm{~km}^{3} /$ an et cumulent ensemble $60 \%$ du total mondial (tableau 2).

Contrairement à ce que croient et affirment certains, qui focalisent l'analyse sur l'unique secteur de l'alimentation en eau potable, les pays développés et industrialisés ne sont donc pas seuls à utiliser beaucoup d'eau, ni dans l'absolu ni par habitant. La Chine et l'Inde ensemble utilisent plus d'eau que les États-Unis, l'Union Européenne, le Japon et la Russie réunis...

Un tableau de ces prélèvements agrégeant les pays par grands espaces économiques, en atténuant de ce fait les incertitudes de nombreuses statistiques nationales, mais distinguant les principaux secteurs d'utilisation, a pu être dressé moyennant quelques hypothèses (Tab. 3). Il fait bien ressortir

\section{4. - BESOINS ET DEMANDES EN EAU : NE PAS CONFONDRE}

- Le besoin en eau est un concept théorique déterminé par les nécessités et les objectifs de l'activité qui l'engendre et par la relation d'efficacité entre les usages de l'eau - en quantité et qualité données - et les résultats. II est donc exprimé le plus souvent de manière "unitaire (par habitant, par hectare irrigué, par unité de produit...). Le besoin en eau a un caractère normatif (c'est une référence pour évaluer la demande présente) et prévisionnel (c'est une base de projet). II est indépendant de l'offre.

- La demande en eau est un fait réel observable, déterminé non seulement par les nécessités de l'activité utilisatrice, mais aussi par l'influence de l'offre qu'elle rencontre : l'offre de la nature - la ressource - aussi bien que celle d'un secteur intermédiaire de production-distribution. La demande peut alors être supérieure au besoin (en cas d'offre surabondante et très accessible) ou inférieure (en cas d'offre rare et chère, suivant les critères des usagers), en quantité comme en qualité. La demande est en somme la transposition et l'expression du besoin dans le réel.

Tableau 2. Prélèvements dans les pays les plus utilisateurs du monde..

\begin{tabular}{|c|c|c|}
\hline & $\begin{array}{l}\text { Population } \\
\text { en M hab }\end{array}$ & $\begin{array}{c}\text { Prélèvements pour toutes } \\
\text { utilisations en } \mathrm{km}^{3 / a n}\end{array}$ \\
\hline Inde (1990) & 849,50 & 552 \\
\hline Chine (1988) & 1088,00 & 500 \\
\hline U.S.A. (1990) & 250,90 & 467 \\
\hline Pakistan (1990) & 114,00 & $242 *$ \\
\hline Russie (1990) & 146,00 & $136 \star$ \\
\hline Union Européenne (1985-90),en comparaison & 350,00 & 245 \\
\hline
\end{tabular}




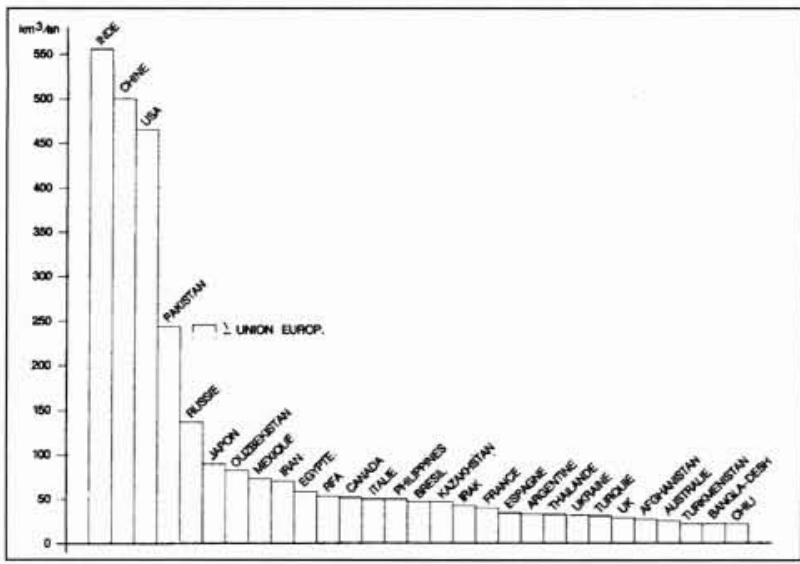

1. Classement des 29 pays utilisant plus de $20 \mathrm{~km}^{3}$ d'eau par an (1990 ou année proche).

les différences de structure des demandes suivant ces ensembles (Fig. 2), ainsi que le poids largement prédominant de l'irrigation. Il propose aussi une estimation des consommations nettes (volumes d'eau non restitués après usage : évapo-transpirés ou rejetés en mer). Plus que les prélèvements bruts, ces consommations définissent en effet la pression quantitative sur les ressources, additives à toutes échelles, tandis que les eaux prélevées et retournées aux eaux continentales après usage peuvent être mobilisées à nouveau.

\subsection{Utilisation d'eau par habitant}

Il est instructif par ailleurs de rapporter ces volumes annuels aux populations, pour mesurer grossièrement la variété des demandes dans le monde et pour rendre les demandes en eau de chaque pays mieux comparables, en faisant ressortir les incidences des deux autres facteurs (Fig. 3). Malgré le fort nivellement opéré par un tel ratio à l'échelle de chaque pays, surtout pour les plus peuplés, les demandes en eau par tête calculées varient dans une gamme très étendue : la moyenne mondiale actuelle, de l'ordre de $600 \mathrm{~m}^{3} / \mathrm{an}$ par habitant (dont environ $50 \mathrm{~m}^{3} / \mathrm{an}$, ou $135 \mathrm{l} /$ jour, d'eau potable), n'est qu'un point de repère théorique dans une gamme allant de moins de 50 à plus de $2000 \mathrm{~m}^{3} / \mathrm{an}$. On peut en déduire une échelle des utilisations d'eau en quantité, suivant laquelle les pays peuvent être classés indépendamment de leur population (Tab. 5) et les utilisations d'eau des pays

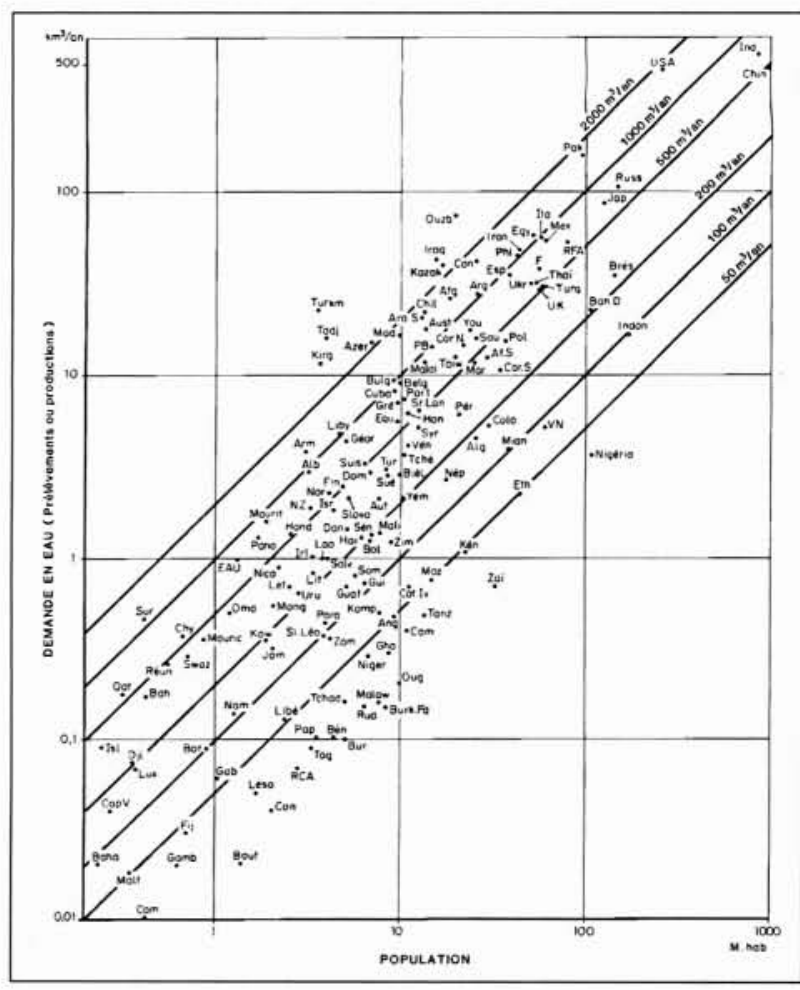

3. Relations entre les demandes en eau et les populations des pays du monde (années 80-90).

peuvent être appréciées comme relativement fortes ou faibles par rapport à celle d'une classe médiane (Tab. 4).

Une géographie macro-économique des demandes en eau peut s'esquisser sur cette base (Fig. 4). La population mondiale, on le voit, est répartie à peu près également entre les pays à demande en eau moyenne par habitant respectivement supérieure et inférieure à $500 \mathrm{~m}^{3} / \mathrm{an}$.

\subsection{Qui utilise le plus ou le moins d'eau ?}

Les demandes par habitant les plus fortes se trouvent dans les pays où l'irrigation pèse très largement dans les utilisations (Turkménistan et autres pays d'Asie centrale de l'ex-URSS, Iraq, Pakistan, Madagascar, Iran, Égypte) aussi bien que dans des pays industrialisés très développés (États-Unis, Canada), les deux facteurs pouvant se combiner (États-Unis, Espagne,
2. Répartition des demandes en eau actuelles par secteur d'utilisation dans chaque grande région géopolitique (cf. tableau 3).

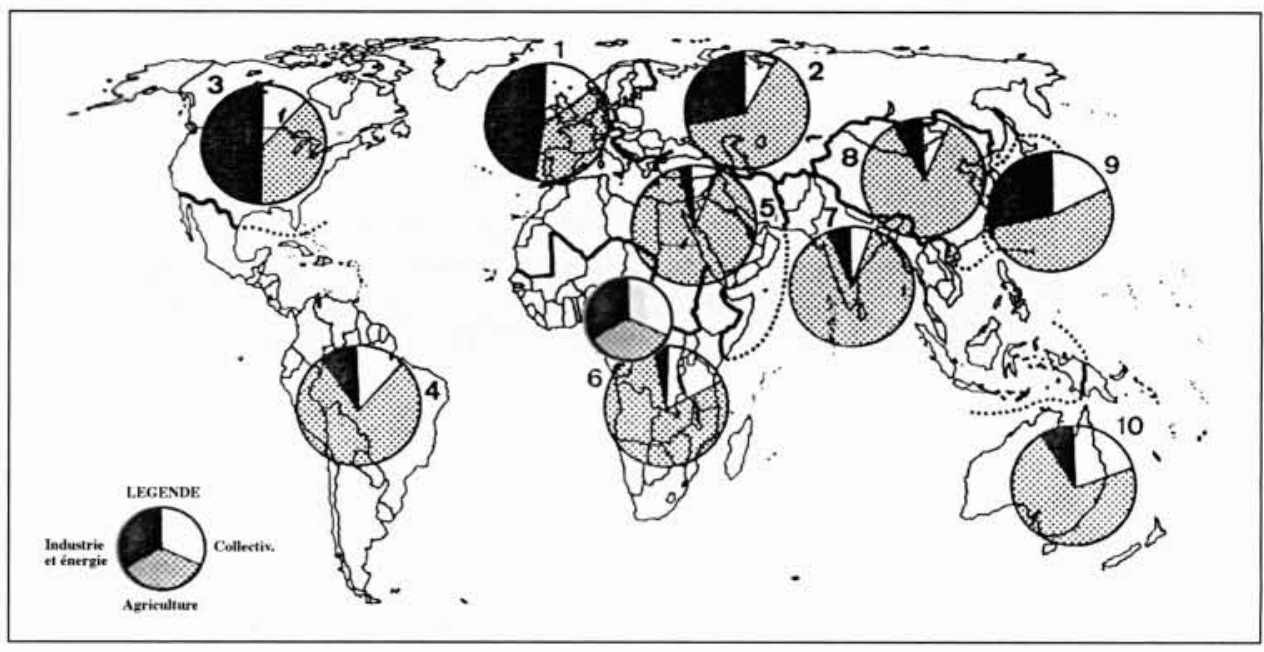




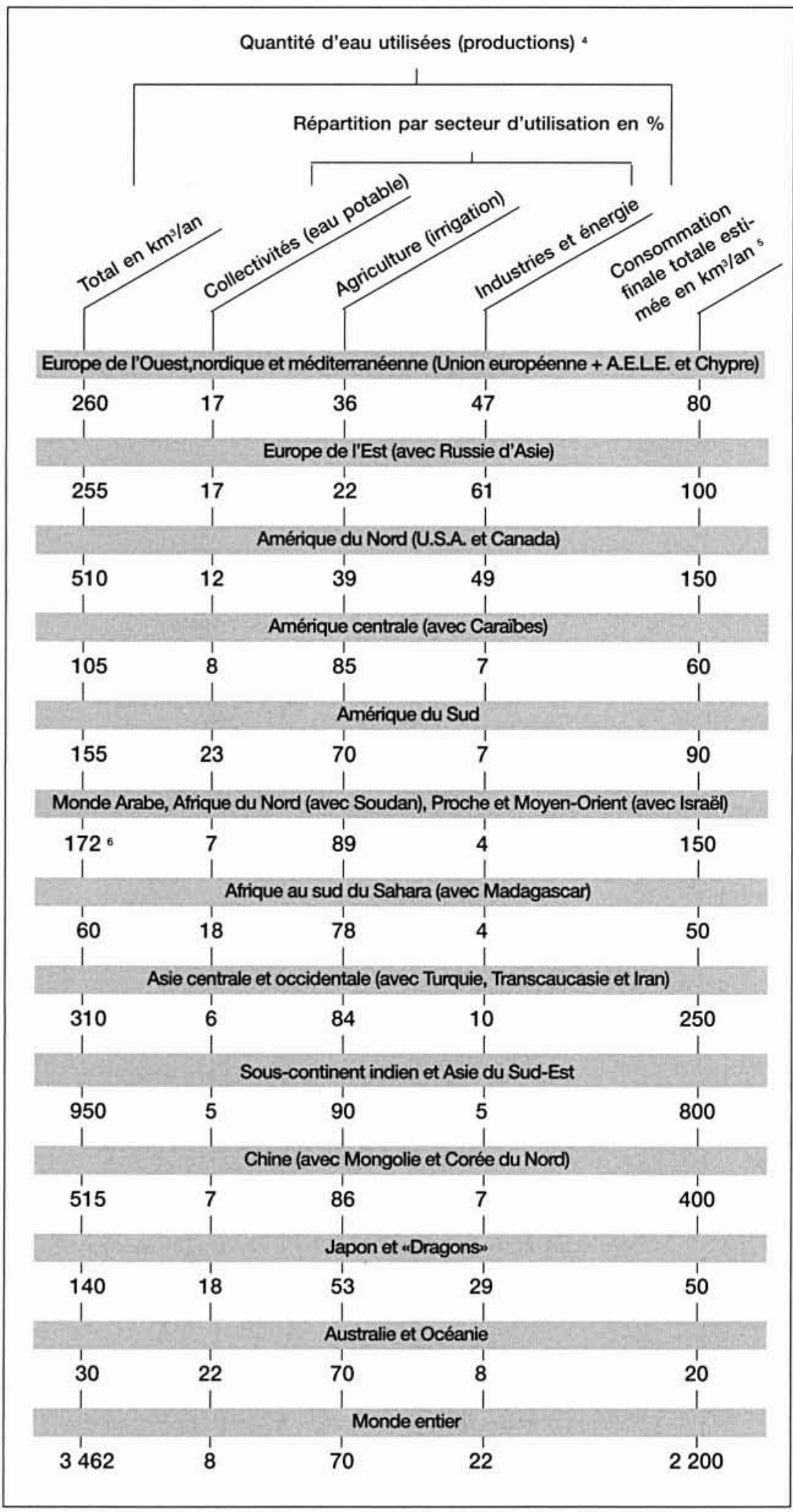

Tableau 3. - Quantités d'eau utilisées dans le monde actuellement (1990 ou année proche).
Argentine, Chili, Australie). Les principaux pays industrialisés où l'irrigation est négligeable, ou non dominante parmi les demandes, se rangent dans la classe des demandes modérées (RFA. France, Japon, Royaume Uni, Russie) aux côtés de pays où le poids des populations réduit le ratio malgré l'importance des irrigations (Chine, Inde).

A l'opposé, les demandes les plus faibles se trouvent soit dans des îles à ressources très limitées (Bahamas, Malte), soit en pays peu développés en zone tropicale humide (Afrique centrale, Insulinde). Ces demandes sont aussi plafonnées par la faiblesse des ressources (ou des déficiences d'aménagement) dans certains pays en zone aride, malgré les nécessités d'irrigation (Algérie, Tunisie, Israël). Il va sans dire que, dans le cas de pays très étendus diversifiés en climat et en densité de population (États-Unis, Chine, Russie...), le calcul d'un index unique masque de fortes différences régionales internes.

Ces demandes par habitant ne sont pas stables : elles tendent à croître ou à décroître suivant les pays - on le verra -, car les populations et les demandes en eau totales évoluent en partie de manières indépendantes.

\section{III — UTILISATION D'EAU ET NIVEAUX DE DÉVELOPPEMENT}

\subsection{Les pays riches utilisent-ils plus d'eau que les pays pauvres?}

Les structures des demandes dépendent largement d'autres facteurs que du niveau de développement socio-économique, notamment du climat. De ce fait, on n'observe pas entre le volume des demandes en eau totales et un indicateur de développement tel que le P.N.B. ${ }^{7}$, de relation aussi nette que celle connue, par exemple, entre le P.N.B. et la consommation d'énergie, qui fait apparaître une élasticité positive. La comparaison tentée entre les quantités d'eau prélevées annuelles et les P.N.B. d'un grand nombre de pays (années de la décennie 80), illustrée par la figure 5 , le met en évidence. Les quantités d'eau utilisée par unité de P.N.B., exprimées en $\mathrm{m}^{3}$ par dollar, varient dans le rapport de 1 à 1000 (de 10 litres à $10 \mathrm{~m}^{3}$ ). Les pays développés n'utilisent pas plus d'eau, en moyenne, que les pays en développement.

La dispersion des grandeurs de prélèvement à P.N.B. égal - et réciproquement - paraît plutôt assez révélatrice du peu de relation entre les quantités d'eau prélevées pour satisfaire les demandes des différentes branches d'activité économique et les contributions de celles-ci à la formation du P.N.B. Il n'est que de comparer sous les deux angles l'agriculture irriguée, notamment à diverses branches d'industrie à forte

\footnotetext{
En prenant cet indicateur pour ce qu'il vaut... et en sachant qu'il n'a san doute pas la même signification pour tous les pays et qu'il prend inégale-
} ment en compte les activités utilisatrices d'eau non marchande.

\footnotetext{
${ }^{4}$ Sans compter l'évaporation des retenues.

${ }^{5} \mathrm{Y}$ compris l'évaporation des retenues.

${ }^{6} \mathrm{Y}$ compris des réutilisations et des productions d'eau non conventionnelles
} 


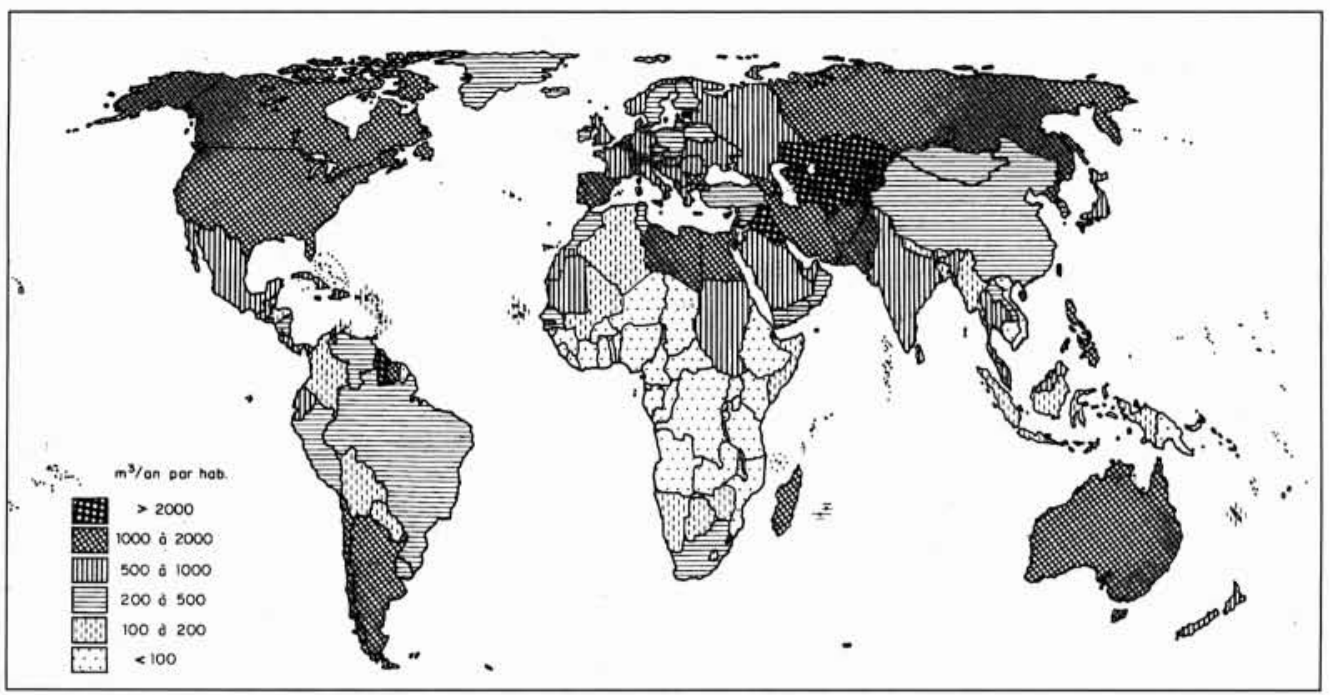

4. Pays classé suivant les quantités d'eau utilisées (prélèvements) par habitant pour toutes utilisations (1990 ou année proche).

"valeur ajoutée". La figure 5 montre bien que, dans une colonne à P.N.B. sensiblement égal, les prélèvements en eau totaux sont d'autant plus élevés que l'agriculture irriguée pèse plus dans la demande en eau ${ }^{8}$.

Cette dispersion reflète donc bien la diversité des structures de demande en eau à un même niveau de développement économique. Par contre, la comparaison entre pays de structure similaire fait mieux ressortir la relation entre les prélèvements et le niveau de développement : par exemple, le groupe de pays à important secteur d'irrigation traditionnelle à demande en eau très prédominante (Cf. Soudan, Égypte, Pakistan, Inde) ou celui des pays industrialisés où l'agriculture est peu ou non irriguée et ne contribue que pour une part minoritaire à la formation du P.N.B.

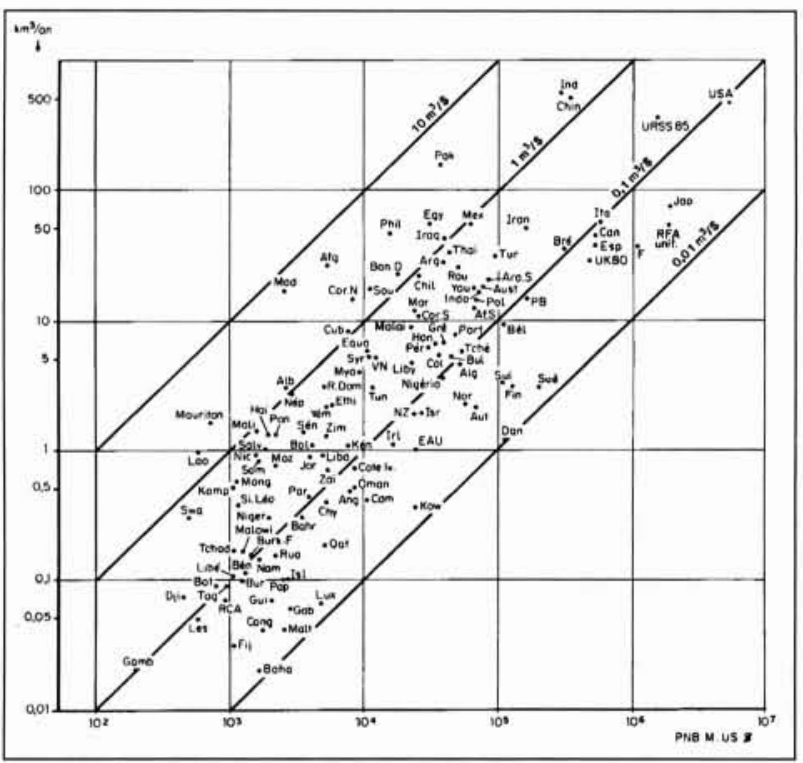

5. Relation entre les demandes en eau totales et les P.N.B. en différents pays (années $80-90$; pour chaque pays les dates de référence des deux variables sont cohérentes).

${ }^{8}$ Naturellement, la relation est aussi dispersée en opérant avec les volumes d'eau et les P.N.B. par habitant, même si les grandes différences de population modifient les positions des pays.

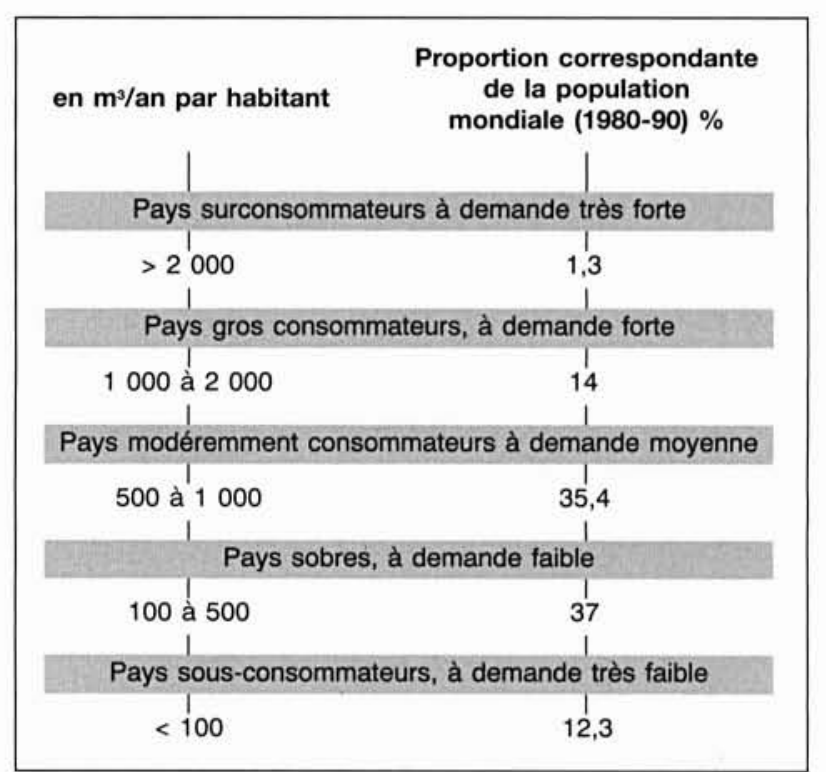

Tableau 4. - Appréciation des consommations en eau.

Plus instructive est l'analyse des évolutions entre les prélèvements en eaux totaux et le P.N.B. en chacun des pays pour lesquels on dispose de données adéquates, depuis une quinzaine d'années (Fig. 6).

\section{Quelle variable explique l'autre ?}

Dans la plupart des pays industrialisés, il y a bien eu une relation positive, mais la croissance des prélèvements en eau a été beaucoup plus lente que celle du P.N.B. et dans certains cas une relation inverse s'est amorcée (États-Unis) du fait d'une décroissance des prélèvements. Aussi, le volume prélevé annuel par \$ de P.N.B. a-t-il diminué en moyenne de 3 à 5 fois, quel que soit son niveau initial en 1975 (Fig. 6), assez régulièrement (États-Unis, Japon, Royaume Uni, Suède...) ou avec un palier et une légère remontée entre 1980 et 1985 (France, RFA, Espagne...) du fait de régressions des P.N.B. La croissance économique est donc très loin d'avoir fait croître d'autant les utilisations d'eau et elle tend à être sans effet sur celle-ci à partir d'un certain niveau de développement. 


\begin{tabular}{|c|c|c|c|}
\hline \multirow{5}{*}{ Plus de 2000} & Turkménistan & 88 & 6859 \\
\hline & Ouzbékistan & 90 & 3682 \\
\hline & Iraq & 85 & 2655 \\
\hline & Kazakhstan & 90 & 2580 \\
\hline & Azerbaïdjan & 88 & 2179 \\
\hline \multirow{12}{*}{1000 à 2000} & États-Unis & 90 & 1873 \\
\hline & Pakistan & 90 & 1766 \\
\hline & Canada & 90 & 1760 \\
\hline & Chili & 90 & 1660 \\
\hline & Afghanistan & 87 & 1436 \\
\hline & Australie & 90 & 1425 \\
\hline & Madagascar & 90 & 1377 \\
\hline & Iran & 85 & 1090 \\
\hline & Argentine & 76 & 1087 \\
\hline & Égypte & 92 & 1073 \\
\hline & Libye & 90 & 1046 \\
\hline & Bulgarie & 85 & 1033 \\
\hline \multirow{16}{*}{500 à 1000} & Pays-Bas & 86 & 994 \\
\hline & Russie & 90 & 919 \\
\hline & Espagne & 92 & 882 \\
\hline & Mexique & 90 & 804 \\
\hline & Italie & 90 & 782 \\
\hline & Syrie & 90 & 768 \\
\hline & Arabie Saoudite & 85 & 766 \\
\hline & Japon & 87 & 731 \\
\hline & France & 94 & 705 \\
\hline & RFA unif. & 90 & 658 \\
\hline & Inde & 90 & 650 \\
\hline & Soudan & 90 & 615 \\
\hline & Ukraine & 88 & 595 \\
\hline & Nouzelle-Zélande & 90 & 571 \\
\hline & Turquie & 90 & 544 \\
\hline & Royaume-Uni & 80 & 507 \\
\hline \multirow{8}{*}{200 à 500} & Maroc & 90 & 462 \\
\hline & Suède & 90 & 458 \\
\hline & Pologne & 90 & 404 \\
\hline & Chine & 90 & 395 \\
\hline & Israël & 94 & 344 \\
\hline & Afrique du Sud & 90 & 328 \\
\hline & Brésil & 90 & 270 \\
\hline & Tunisie & 95 & 248 \\
\hline \multirow{5}{*}{100 à 200} & Sénégal & 90 & 185 \\
\hline & Algérie & 90 & 180 \\
\hline & Mali & 90 & 142 \\
\hline & Zimbabwe & 87 & 130 \\
\hline & Malte & 90 & 111 \\
\hline \multirow{12}{*}{ moins de 100} & Botswana & 80 & 100 \\
\hline & Indonésie & 87 & 96 \\
\hline & Côte d'Ivoire & 87 & 68 \\
\hline & Gabon & 87 & 51 \\
\hline & Ethiopie & 87 & 48 \\
\hline & Kenya & 87 & 48 \\
\hline & Togo & 87 & 40 \\
\hline & Nigéria & 90 & 37 \\
\hline & Cameroun & 87 & 30 \\
\hline & Burkina Faso & 90 & 38 \\
\hline & Congo (Zaire) & 80 & $\sim 10$ \\
\hline & Bouthan & 90 & $\sim 5$ \\
\hline
\end{tabular}

Tableau 5. - Sélection de pays classés suivant leurs demandes en eau contemporaines (pour toutes utilisations) par habitant. par ordre décroissant).
Dans les pays moins développés, les évolutions ont été beaucoup plus variées. Généralement, en décroissance marquée avant 1980, dans certains cas à partir de valeurs très élevées (Égypte, Inde, Iraq...) - de 10 à 15 fois supérieures à celles des pays industrialisés - les volumes prélevés par $\$$ de P.N.B. ont ensuite tantôt continué à diminuer (Inde, Iran), avec parfois des paliers ou des stabilisations indiquant des évolutions parallèles des utilisations d'eau et du P.N.B. (Égypte, Iraq, Maghreb, Turquie), tantôt au contraire ils se sont accrus, parfois fortement dans des pays pétroliers (Arabie Saoudite, Libye) où, à l'évidence, l'enrichissement a plus été le facteur du développement des utilisations d'eau (notamment en irrigation) que son résultat.

\subsection{Les demandes et les consommations en qualité sont tout aussi diverses}

Sont à bien distinguer ici : les demandes en eau de différentes qualités requises par les usages (encadré 5), les consommations en qualité (usures) par ces usages et les consommations des "ressources en qualité" (les dégradations) qui résultent finalement des retours d'eau usée dans les eaux du milieu.

Les unes comme les autres dépendent d'abord des usages, très inégalement exigeants et différemment "usants" (ils ne dégradent pas les mêmes variables de qualité, ni aux mêmes degrés), sans lien direct le plus souvent avec les quantités d'eau en service. Chaque secteur d'utilisation mettant en jeu des usages variés, sa demande en qualité tend à s'aligner sur le besoin de l'usage le plus exigeant, tandis que sa consommation additionne celles de chacun des usages. Par ordre d'usure de qualité (c'est-àdire de concentration des rejets) décroissante, les utilisations d'eau se classent en gros comme suit : industries (hors refroidissement), utilisations domestiques, irrigation, refroidissement industriel et énergétique.

Les consommations finales des ressources en qualité dépendent en outre à la fois des taux d'épuration des rejets et des capacités diluantes et régénératrices des milieux récepteurs des retours d'eau.

Dans une certaine mesure la grandeur des populations non desservies par des installations d'épuration des eaux usées pourrait servir d'indicateur des consommations en qualité des collectivités, notamment par les utilisations domestiques - encore que les charges rejetées par habitant soient loin d'être uniformes dans le monde... Cet indicateur peut être calculé pour les pays développés (par exemple dans les pays de l'OCDE, en 1985, 320 millions d'habitants, $44 \%$ de la population, n'étaient pas desservis par des stations d'épuration - source OCDE 1989 -), mais les données manquent dans la plupart des autres pays où l'on peut augurer que cette proportion soit encore plus forte. 


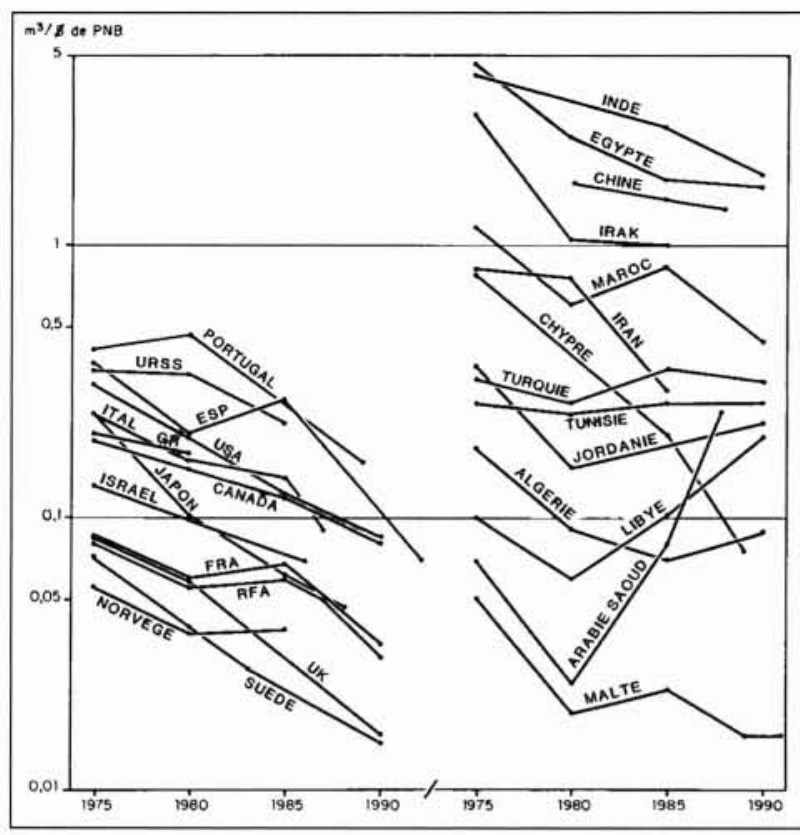

6. Evolution des rapports entre "prélèvements en eau totaux et P.N.B.", en m³/US\$, de 1975 à 1990 en différents pays. Ces deux variables étant peu dépendantes, la décroissance fréquente du ratio indique surtout que les PNB sont plus croissants que les quantités d'eau prélevées.

\subsection{Quelle part des ressources exploite-t-on et consomme-t-on ?}

Les volumes d'eau utilisés à présent sont encore à comparer aux ressources naturelles et renouvelables. Bien que les différences de taille entre les pays faussent en partie les comparaisons, de la double diversité des ressources et des demandes en eau, estimées sommairement par pays entier, résulte une extrême variété de situations. La gamme des indices d'exploitation (prélèvements totaux rapportés au flux moyen de ressources naturelles, intérieures ou extérieures) est très étendue, allant de moins de $1 \%$ à plus de $100 \%$ dans quelques pays qui tirent sur les réserves (Fig. 7). La variété des quantités utilisées est peu corrélée avec celle des ressources offertes : des pays riches en eau sont tantôt dépensiers, tantôt économes (parfois par force, faute de moyens - exemple : Afrique centrale -, mais aussi par le peu de besoin) ; des pays moyennement pourvus sont très inégalement demandeurs (surtout suivant le poids de l'agriculture irriguée) ; des pays pauvres en eau en utilisent tantôt cependant beaucoup (plusieurs pays arabes), tantôt très peu (Sahel). La faiblesse des ressources contribue toutefois à plafonner les demandes dans les pays économiquement pauvres.

Plus que les prélèvements dont une partie, retournée après usage aux eaux continentales, réintègre les ressources et peut "resservir", ce sont les consommations finales qui exploitent quantitativement les ressources à l'échelle des grandes

\section{5. - DE QUELLES QUALITÉS D'EAU A-T-ON BESOIN ?}

Tout besoin en qualité d'eau est nécessairement :

- Spécifique à un usage défini.

Chaque usage met en jeu des fonctions particulières de l'eau auxquelles correspondent des "paramètres de qualité propres : teneurs en différentes substances et en microorganismes pour l'alimentation humaine, salinité pour l'irrigation, dureté pour la production de vapeur, température pour le refroidissement...

- Relatif à une efficacité désirée.

Entre les grandeurs des caractéristiques de qualité de l'eau et les résultats de sa mise en usage, il n'y a pas de relation en "tout ou rien" mais une variation continue de rendement qui appelle un choix. Cette efficacité n'est généralement pas indépendante de la quantité d'eau utilisée.

Une norme de qualité n'est donc pas un absolu, mais la traduction conventionnelle et simplificatrice d'un objectif d'efficacité (usages agricoles et industriels), de sécurité sanitaire ou encore de confort (usages domestiques), qui facilite son expression réglementaire et les contrôles (normes de potabilité), mais ne doit pas faire perdre de vue son caractère relatif. L'objectif des normes peut différer aussi suivant les variables considérées : en matière de potabilité, les normes de toxicité n'admettent aucune défaillance sans exposition à un risque, alors que les normes de confort peuvent tolérer une certaine fréquence de défaillance.

Lorsque de l'eau doit servir à différents usages, ce qui est le cas général des utilisations domestiques et industrielles, c'est l'usage le plus exigeant en qualité (pour chaque variable) qui détermine le besoin en qualité commun. Cela entraîne certes une qualité superflue pour les autres usages (par exemple le lavage d'automobile ou l'arrosage à l'eau potable : on sait que moins de $10 \%$ des besoins en eau domestiques nécessitent en toute rigueur de l'eau potable). Des distributions d'eau séparées ou des traitements d'eau fractionnés sélectifs, concevables en théorie pour offrir à chaque usage l'eau de qualité nécessaire et suffisante (on débat depuis longtemps du "double réseau"), sont cependant peu praticables, hors de cas particuliers : les filières de réutilisation où il est possible d'enchaîner les usages par ordre de besoins en qualité décroissants afin de minimiser les traitements intercalaires. Cela se pratique déjà dans les grands immeubles au Japon.

La géographie des consommations en qualité des eaux ne reflète ainsi qu'en partie celle des utilisations (Fig. 2), qu'il conviendrait de pondérer par les quantités correspondantes (Tab. 3). La répartition des consommations finales dépendra aussi de celle des efforts de traitement des eaux usées, dont l'ampleur est largement subordonnée au niveau de développement socio-économique.

S'il n'est pas possible de dresser un tableau similaire à celui des quantités d'eau utilisées, du moins peut-on présumer que les principales consommations en qualités doivent être concentrées :

- dans les pays industrialisés, surtout ceux où sont particulièrement développés la chimie, la pétrochimie, la papeterie..., et ceux où la gestion des déchets est défectueuse ;

- dans les zones à forte urbanisation des pays peu développés ;

- dans les zones à irrigation insuffisamment accompagnée de drainage, facteur de salinisation en régions arides ou semi-arides. 


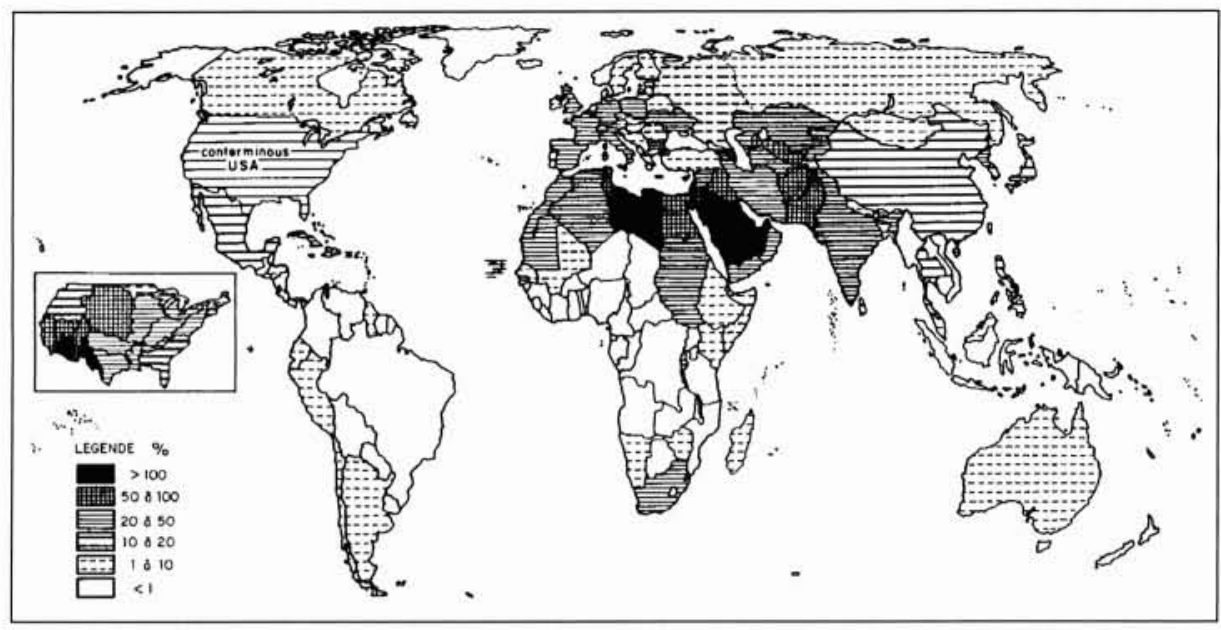

7. Proportions exploitées actuelles des ressources en eau naturelles et renouvelables : pays classés par "indice d'exploitation" (années 80-90). (Conterminous USA : Alaska, Hawaï et Porto-Rico non compris)

régions, bassins et pays. Malgré les incertitudes de leur estimation, beaucoup plus calculée d'après les cœfficients statistiques moyens et des hypothèses sur les parts des rejets déversés en mer, que basée sur des mesures, la géographie des consommations finales est significative. C'est, à l'évidence, le reflet de la répartition des utilisations les plus consommatrices, celles des irrigations pour l'essentiel. Aussi, plus de la moitié des consommations finales se situe en Asie. La Chine et l'Inde à elles seules consomment le quart du total mondial. Ces consommations auraient déjà fait croître de 3 à $4 \%$ l'évaporation des terres émergées.

Plus significatif aux échelles nationales et régionales que les indices d'exploitation, les indices de consommation finale (consommations finales rapportées au flux moyen de ressources naturelles) des différents pays mettent en évidence autant de variétés et de contrastes.

Les géographies des indices d'exploitation (Fig. 7) et de consommation finale montrent concurremment que les pressions quantitatives majeures sur les ressources en eau se situent d'abord dans le monde arabe (Afrique septentrionale et Moyen-Orient) et en Asie centrale et méridionale, puis secondairement dans l'Ouest de l'Amérique du Nord, en reflétant assez bien la carte de l'aridité.

Naturellement, comparer les prélèvements et les consommations finales aux seules ressources naturelles et à leurs seuls flux moyens ne donne qu'une idée sommaire et sous-estimée des pressions humaines sur les eaux du milieu, car celles-ci sont très inégalement exploitables suivant leur répartition dans l'espace et leur variabilité, et suivant les efforts (donc les coûts) que leur mobilisation nécessite. Des parts variées, mais rarement minimes, des ressources naturelles sont en pratique inexploitables. L'exploitabilité de ces ressources n'est certes pas définissable avec unicité et constance, car les critères varient suivant les utilisations et peuvent évoluer à mesure que les disponibilités se raréfient. Les indices d'exploitation et de consommation précédents, rapportés aux ressources naturelles moyennes et globales par pays, n'en minimisent pas moins les pressions réelles, en masquant les incidences locales et conjoncturelles ; ce sont seulement des indicateurs macroscopiques de situation moyenne.

\subsection{Quelles ressources en eau exploite-t-on ?}

La répartition des prélèvements en eau par type de ressource (superficielle régulière ou irrégulière, souterraine renouvelable ou non renouvelable) se plie largement aux structures et aux poids relatifs de ces ressources propres à chaque pays. Mais elle dépend aussi en partie de la structure des demandes plus ou moins concentrées ou dispersées, collectives ou individuelles.

Globalement dominante $(80 \%$ des prélèvements mondiaux), l'exploitation des eaux superficielles est développée le plus dans les pays humides à systèmes fluviaux denses et abondants ou dans les pays arides à fleuve exogène formant l'essentiel des ressources (Égypte, Iraq, Kazakhstan...). Elle fournit la plus grande partie des eaux utilisées pour l'irrigation et la desserte des grandes villes, la totalité des eaux de refroidissement et une part majeure des eaux industrielles.

\begin{tabular}{|lcc|}
\hline Utilisations & $\begin{array}{c}\text { Eaux superficielles } \\
\text { monde entier }\end{array}$ & $\begin{array}{c}\text { Eaux souterraines } \\
\%\end{array}$ \\
\hline Alimentation des collectivités & 50 & 50 \\
\hline Irrigation & 80 & 20 \\
\hline Industries non desservies & 60 & 40 \\
\hline Centrales thermiques* & $\sim 100$ & $\varepsilon$ \\
\hline
\end{tabular}

*à refroidissement par eau douce

Tableau 6. - Proportions d'eaux superficielles et souterraines utilisées dans chaque secteur d'utilisation. 


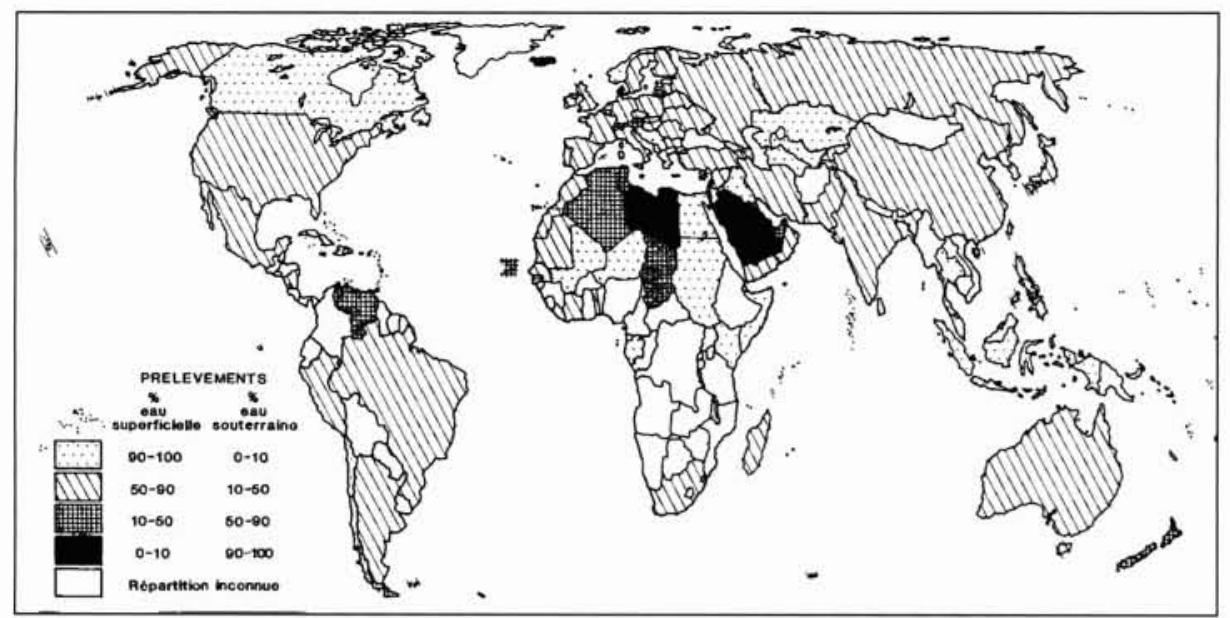

8. Répartition des prélèvements en eau, dans chaque pays, suivant le type de ressource exploitée.

L'exploitation des eaux souterraines est prédominante en quelques pays à faibles ressources superficielles, principalement lorsqu'il s'agit de ressources non renouvelables (Arabie, Libye). Elle n'est cependant pas négligeable dans les pays tempérés ou tropicaux à ressources en eau souterraine notables, où elle couvre une partie appréciable des demandes en eau dispersées : alimentation rurale en majorité, irrigation individuelle. Rien qu'en Inde, par exemple, $160 \mathrm{~km}^{3} / \mathrm{an}$ d'eau souterraine sont prélevés actuellement pour irriguer : $35 \%$ de tout le volume d'eau d'irrigation de ce pays, record du monde de l'exploitation d'eau souterraine.

Globalement, les eaux superficielles et les eaux souterraines participent aux approvisionnements des principaux

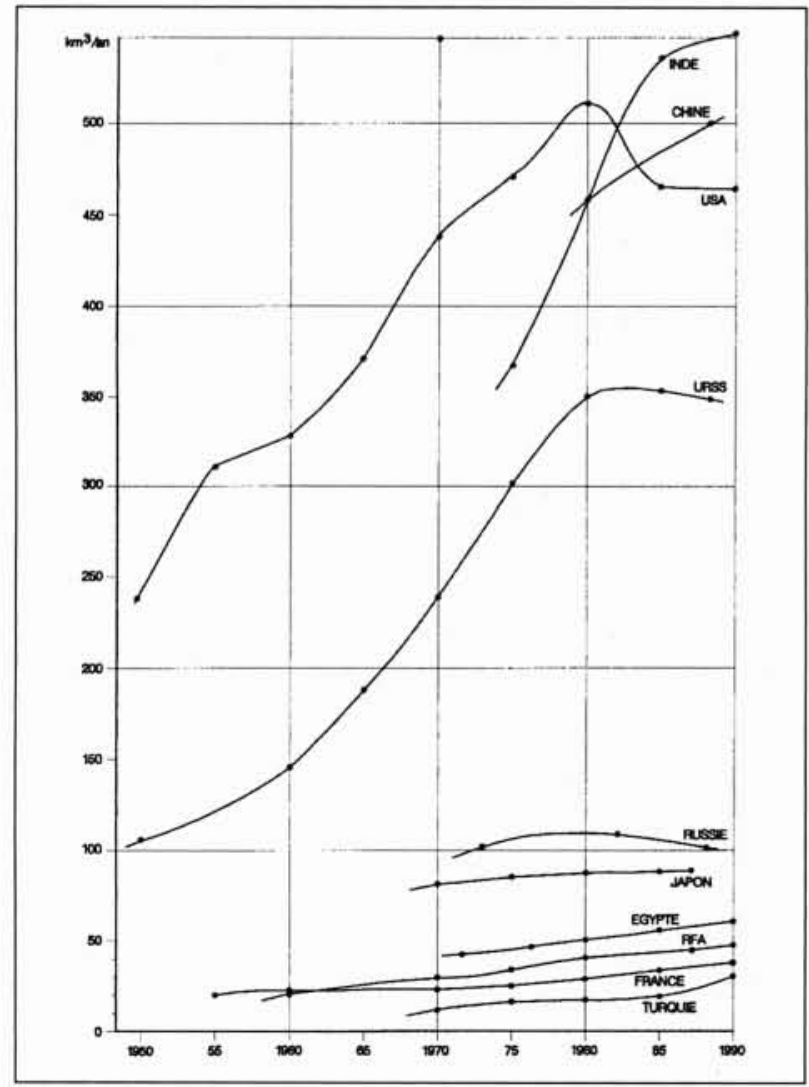

9 . Evolution des demandes en eau totales en quelques pays au cours de la seconde moitié du $\mathrm{XX}^{\mathrm{e}}$ siècle, d'après des sources nationales. secteurs d'utilisation dans les proportions très arrondies inscrites dans le tableau 6.

Mais ces proportions sont très variables suivant les pays (Fig. 8).

\subsection{L'utilisation des eaux s'est fortement accrue au $\mathrm{XX}^{\mathrm{e}}$ siècle}

L'histoire des utilisations d'eau est plus hasardeuse que leur géographie présente. Peu de pays disposent de chroniques chiffrées assez longues et homogènes à ce sujet (la figure 9 en donne quelques exemples). Les estimations passées sont souvent mal raccordables aux évaluations modernes et prennent inégalement en compte tous les secteurs. Les historiques de prélèvements ou de "consommations" établis risquent de traduire autant les progrès des comptages et des méthodes d'estimation que les évolutions réelles. En France, cette évolution ne peut être esquissée, sur la base de quelques estimations très approximatives, qu'à

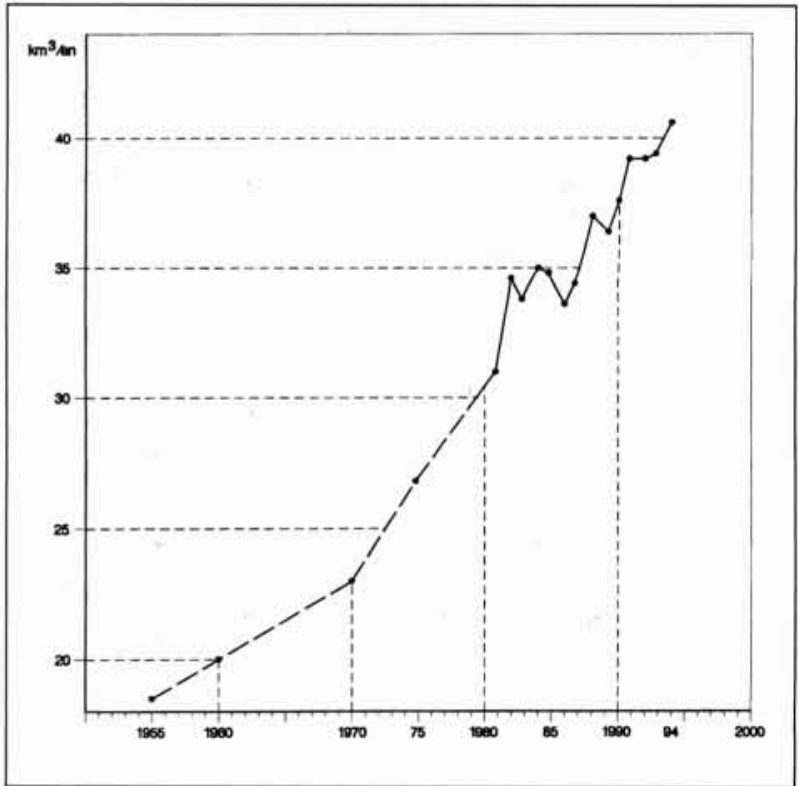

10. Evolution des prélèvements en eau totaux annuels en France de 1955 à 1994 (exhaure des mines, consommations des voies navigables et de l'hydroélectricité non comprises). (Source : de 1955 à 1975 : Commissions de l'eau des Plans et Agences de bassin ; après 1980 : ministère de l'Environnement, 1996). 


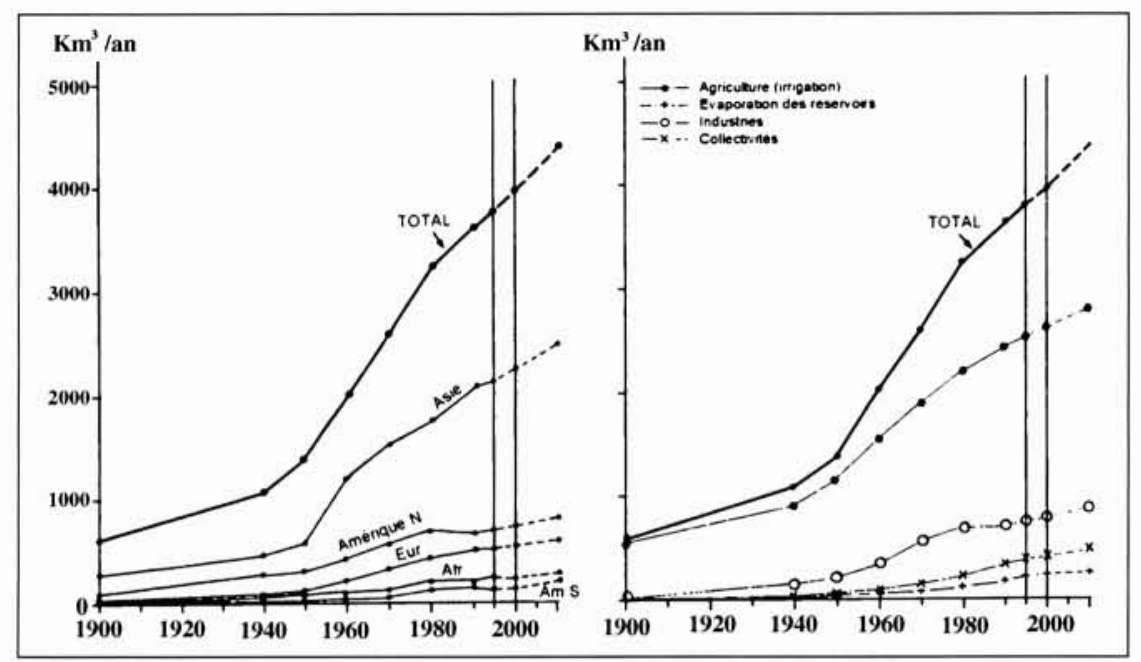

11. Evolution et extrapolation tendancielle des prélèvements en eau mondiaux au $\mathrm{XX}^{e}$ siècle. (Source : Korzun/UNESCO, 1974, 1978 ; Shiklomanov/UNESCO, 1996).

partir des années 50. Elle est mieux connue - et apparaît alors assez irrégulière (peut-être sous l'effet de "biais statistiques" autant que de conjonctures...), depuis une quinzaine d'années (Fig. 10). En 35 ans, les volumes d'eau annuels prélevés auraient doublé.

Quelques reconstitutions globales, par continent et par secteur d'utilisation majeur, ont cependant été tentées, en se basant, comme pour des prévisions, sur l'évolution des principaux facteurs (population, irrigations...) (Fig. 11).

Ces rétrospectives font apparaître une ample croissance globale des prélèvements en eau, encore qu'inégale suivant les continents et les secteurs, en concordance avec leurs états présents.

Globalement, les prélèvements auraient été multipliés par six entre le début du siècle et 1980, et ils dépasseraient aujourd'hui 3000 milliards de $\mathrm{m}^{3} \mathrm{par}$ an. Toutefois, les évolutions reconstituées font apparaître une rupture au début de la seconde moitié du $\mathrm{XX}^{\mathrm{e}}$ siècle (années 50) : passage d'une progression plutôt linéaire à une accélération sensible, la plus forte croissance affectant les demandes de l'irrigation, et celles de l'Asie, à l'évidence liées.

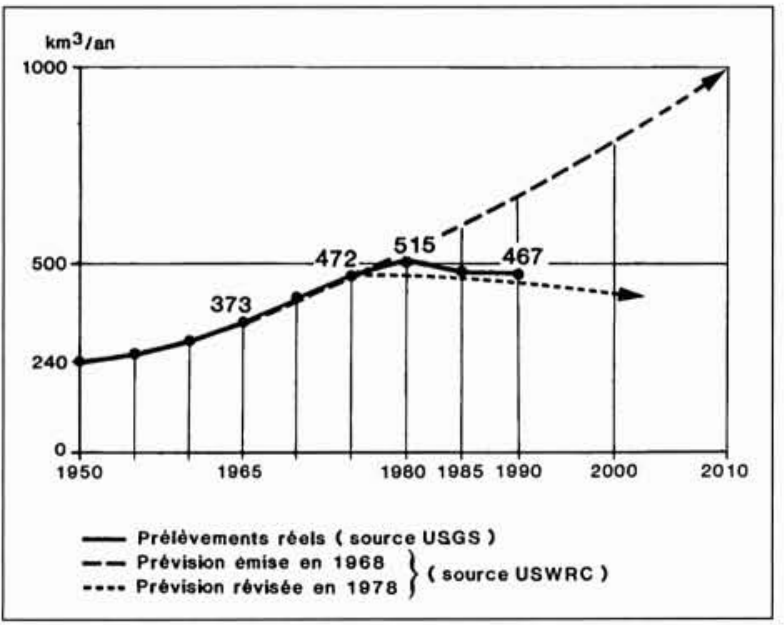

12. Evolution des prélèvements totaux d'eau douce ("offstream use") aux États-Unis depuis 1950 et divergence des prévisions successives. Exemple du danger des projections par extrapolation de tendance.

\section{6. - COMMENT ÉVOLUENT LES DEMANDES EN EAU?}

L'évolution des demandes en eau nationales, régionales ou même mondiales, a le plus souvent été présumée suivre une loi de croissance, comme les populations et les niveaux de développement qui sont ses principaux facteurs.

Les échantillons d'historiques des prélèvements en eau disponibles en quelques pays conduisent plutôt à concevoir une évolution-type en trois phases, suivant une "courbe en S" :

- Stabilité ou faible croissance dans les sociétés préindustrielles, état réalisé actuellement dans les pays "en développement" les plus pauvres et sans irrigation appréciable, quelle que soit leur croissance démographique.

- Croissance forte, parfois accélérée, dans les pays en cours d'industrialisation et dans ceux où la croissance démographique induit celle de l'irrigation, tant que les ressources ne la limitent pas.

- Croissance décélérée, stabilisation ou parfois décroissance dans les pays les plus développés, à population stationnaire, ou dans les pays en développement où les ressources plafonnent la progression des irrigations et où les économies d'eau dans le secteur de l'irrigation peuvent largement compenser la croissance des demandes des autres secteurs.

Ces "historiques" trop globaux, qui semblent corroborer une loi de croissance exponentielle, masquent en fait des évolutions plus différenciées suivant les niveaux de développement. Alors que des croissances ont été significatives dans les pays industrialisés jusqu'à une époque récente, et sont encore fortes dans les pays en développement à démographie galopante et à irrigation en expansion, une décélération s'est amorcée au cours des années 80 dans plusieurs pays développés. Des ruptures de croissance ou même des décroissances sont survenues, mettant en défaut certaines prévisions antérieures, comme aux États-Unis (Fig. 12), ou en France, où les prévisions émises dans les années 60 ou 70 pour l'horizon 85 se sont trouvées démenties par les faits et avaient été très surestimées. 


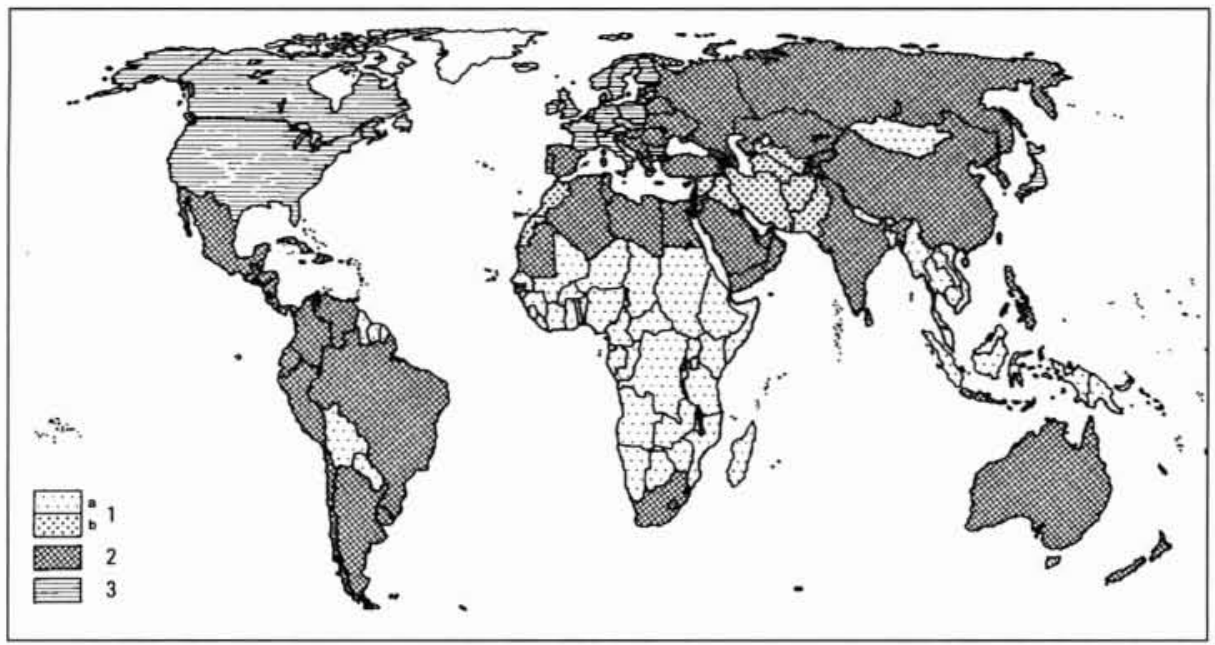

13. Pays classés suivant les tendances actuelles d'évolution des demandes en eau.

1 - Pays en développement à croissance faible : a/du fait de la pauvreté, b/du fait du plafonnement par les ressources.

2 - Pays développés ou en développement à croissance forte.

3 - Pays développés à croissance faible et décélérée, nulle ou négative.

La variété des tendances actuelles observables incite à reconsidérer la "loi d'évolution" des demandes en eau (encadré 6). La croissance globale ne doit pas être confondue avec une croissance universelle : elle n'est en fait que la somme des croissances réelles et de situations stables, ou même de légères décroissances.

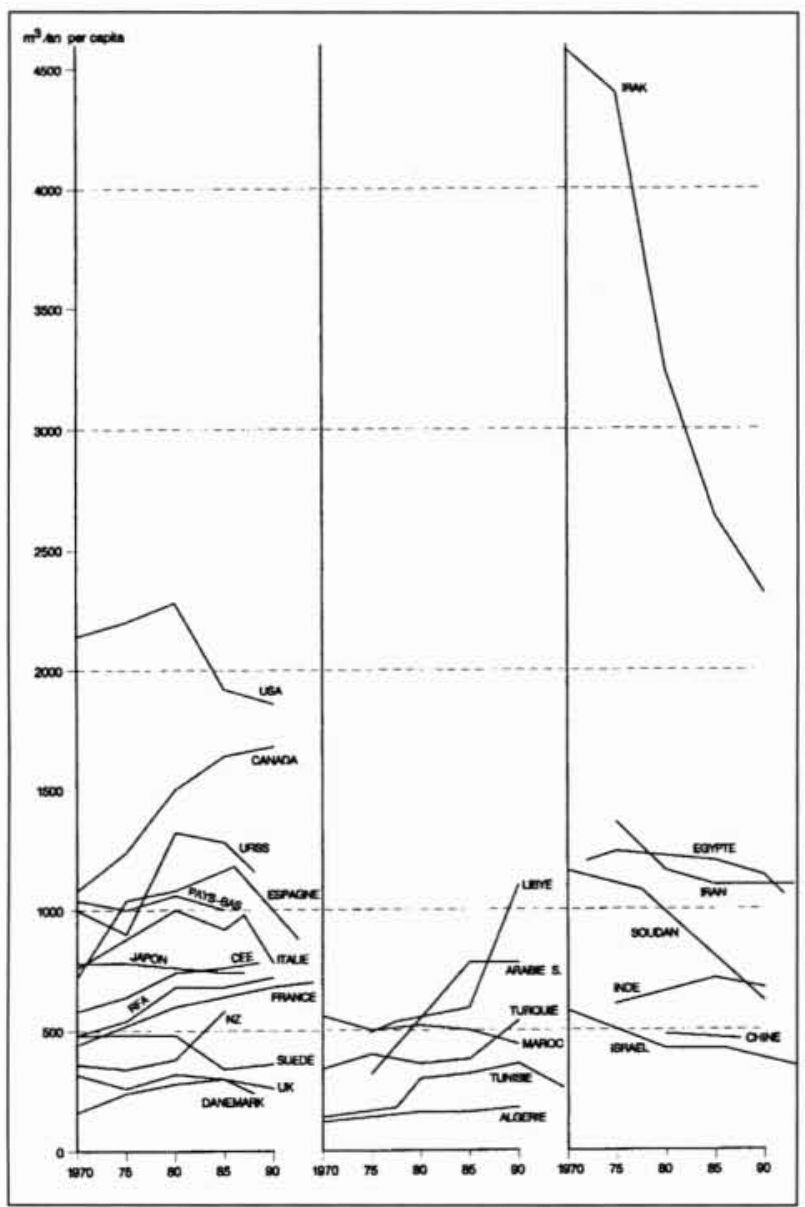

14. Evolution des demandes en eau (pour toutes utilisations) par habitant au cours des deux dernières décennies, en différents pays.

(Sources : O.C.D.E. 1997, W.R.I. 1996, et diverses publications nationales).
Les quantités d'eau utilisées paraissent ainsi ne croître sensiblement qu'au-dessus d'un niveau de développement minimal et jusqu'à un "optimum" au-delà duquel le développement socio-économique ne ferait plus croître les demandes en eau de manière appréciable.

Suivant les tendances présentes, observées ou supputées, d'évolution de leurs prélèvements en eau, la plupart des pays du monde peuvent se ranger dans l'une de ces trois phases (Fig. 13). La géographie de ces tendances est du moins aussi significative que celle des demandes actuelles totales ou par habitant.

Rapportées aux populations, les évolutions des demandes en eau totales ont été aussi fort différentes. Les données historiques disponibles en un certain nombre de pays, pour la période 1970-1990, font apparaître des croissances ou des décroissances des demandes en eau par habitant (Fig. 14).

Dans les pays développés à croissance démographique faible à modérée, ces demandes ont été tantôt croissantes dans des pays où les quantités d'eau utilisées ont grossi plus vite que les populations (Canada, Espagne, RFA ; États-Unis et France jusqu'aux années 80), tantôt décroissantes assez régulièrement (Japon, Suède, Royaume Uni) sous l'effet d'une gestion de l'eau plus économe. Des décroissances se sont amorcées aux États-Unis, en France et dans d'autres pays de l'Union Européenne durant la décennie 80. Dans les pays peu développés aussi s'observent soit des décroissances assez accentuées, mais à partir de niveaux élevés, dans des pays à forte croissance démographique, sans moyens de développer autant les utilisations d'eau (Iraq. Iran, Égypte...), soit des croissances sensibles dans des pays à fort développement de l'irrigation stimulé par des rentes pétrolières (Arabie Saoudite, Libye). Des croissances plus modérées témoignent d'un développement économique plus diversifié (Maghreb), tandis que des amorces de décroissance (Inde, Maroc) traduisent des poussées démographiques plus fortes que la progression des utilisations d'eau.

\subsection{Qu'est-ce qui a le plus fait croître les quantités d'eau utilisées?}

L'explosion démographique au $\mathrm{XX}^{\mathrm{e}}$ siècle est naturellement le facteur primordial de la croissance des utilisations d'eau : à la fois par les demandes en eau potable, surtout celle des agglomérations en forte expansion, qu'elle entraîne, et par les nécessités d'irrigation pour faire face aux besoins 


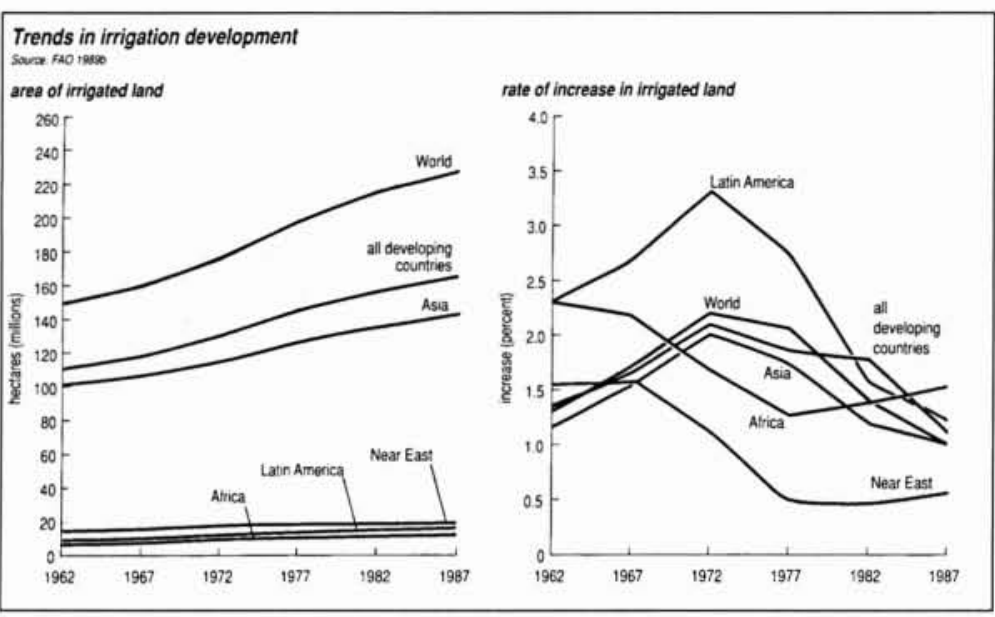

15. Tendance d'évolution des superficies irriguées dans le monde : aires irriguées, à gauche, et taux de croissance annuels des aires irriguées, à droite.

(Source : FAO, 1990).

alimentaires qu'elle induit. Le développement industriel d'une partie du monde est le second facteur.

\subsubsection{L'irrigation}

Les superficies irriguées dans le monde, estimées à environ 48 millions d'hectares au début du siècle selon l'ICID ont quintuplé et approchent aujourd'hui 240 millions d'hectares. Leur croissance a toutefois cessé de s'accélérer depuis les années 70, mais marque une recrudescence en Afrique et au Proche-Orient. Les aires irriguées se seraient encore accrues d'un tiers depuis 20 ans, en passant de 170 millions d'hectares en 1970 à 235 en 1990, selon la FAO, la plus grande partie (plus de $60 \%$ ) et la plus forte croissance se situant en Asie (Fig. 15).

Certes, la croissance des quantités d'eau utilisée pour l'irrigation n'est pas proportionnelle à celle des superficies irriguées. D'abord du fait de la variété des doses d'eau à l'hectare, qui s'échelonnent de 2000 à $20000 \mathrm{~m}^{3} / \mathrm{an}$, voire plus, suivant les climats, les cultures et les sols. Puis de la diversité des "efficiences" - proportions des quantités d'eau apportée aux champs effectivement utilisées par les cultures - suivant les techniques d'arrosage.

Les progrès d'efficience ont pu contribuer à ralentir la croissance des volumes d'eau consacrés à l'irrigation par rapport à celle des superficies. Mais si ces progrès ont été significatifs en quelques pays au cours des dernières années, sous la pression des pénuries d'eau, ils sont restés modestes à l'échelle mondiale où l'on estime que l'efficience moyenne est encore inférieure à $40 \%$. Par exemple, l'irrigation localisée ou "microirrigation" très efficiente, pratiquée sur $71 \%$ des terres irriguées à Chypre, $49 \%$ en Israël et $21 \%$ en Jordanie (pays les plus avancés en cette matière), n'est en service que sur $0,7 \%$ des aires irriguées du monde [17].

Il est encore à remarquer que la croissance des superficies irriguées globales a été renforcée par une croissance des superficies irriguées par habitant jusque vers la fin des années 70 , mais une rupture de tendance s'est produite à la fin de cette décennie : depuis 1978 la superficie irriguée moyenne par habitant dans le monde décroît régulièrement, tombant à 45 hectares pour 1000 en 1989 ; la croissance des populations est désormais plus rapide que celle

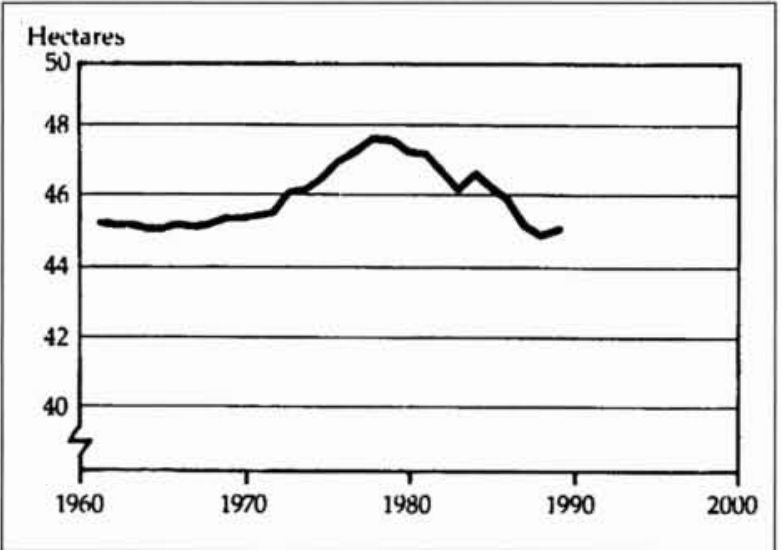

16. Evolution de la superficie irriguée mondiale en hectares par 1000 habitants.

(Source FAO, Production Yearbook 1991).

des aires irriguées dans le monde (Fig. 16) (Source : FAO, Production Yearbook).

\subsubsection{L'urbanisation}

En amplifiant les effets de l'explosion démographique, l'urbanisation galopante a accéléré la croissance des demandes en eau dans le monde, car les populations urbaines requièrent notablement plus d'eau que les ruraux.

La population des villes a décuplé depuis le début du siècle

Tableau 7. - Efficience de l'irrigation suivant les procédés (source FAO, 1988).

\begin{tabular}{|c|c|c|c|}
\hline \multirow{2}{*}{$\begin{array}{c}\text { Mode } \\
\text { d'irrigation }\end{array}$} & \multirow{2}{*}{$\begin{array}{c}\text { Condition } \\
\text { d'application }\end{array}$} & \multicolumn{2}{|c|}{ Efficience en \% } \\
\hline & & sur sol lourd & sur sol léger \\
\hline Aspersion & $\begin{array}{l}\text { - Diurne, avec vent faible } \\
\text { - Nocturne }\end{array}$ & $\begin{array}{l}60 \\
70\end{array}$ & $\begin{array}{l}60 \\
70\end{array}$ \\
\hline $\begin{array}{l}\text { Irrigation } \\
\text { localisée }\end{array}$ & - & 80 & 80 \\
\hline Bassins & $\begin{array}{l}\text { Bien ou mal nivelés et } \\
\text { façonnés }\end{array}$ & 75 à 60 & 60 à 45 \\
\hline $\begin{array}{l}\text { Gravitaires par } \\
\text { rigoles ou à la } \\
\text { planche }\end{array}$ & $\begin{array}{l}\text { Bien ou mal graduée } \\
\text { et dimensionnée }\end{array}$ & 65 à 55 & 50 à 40 \\
\hline
\end{tabular}




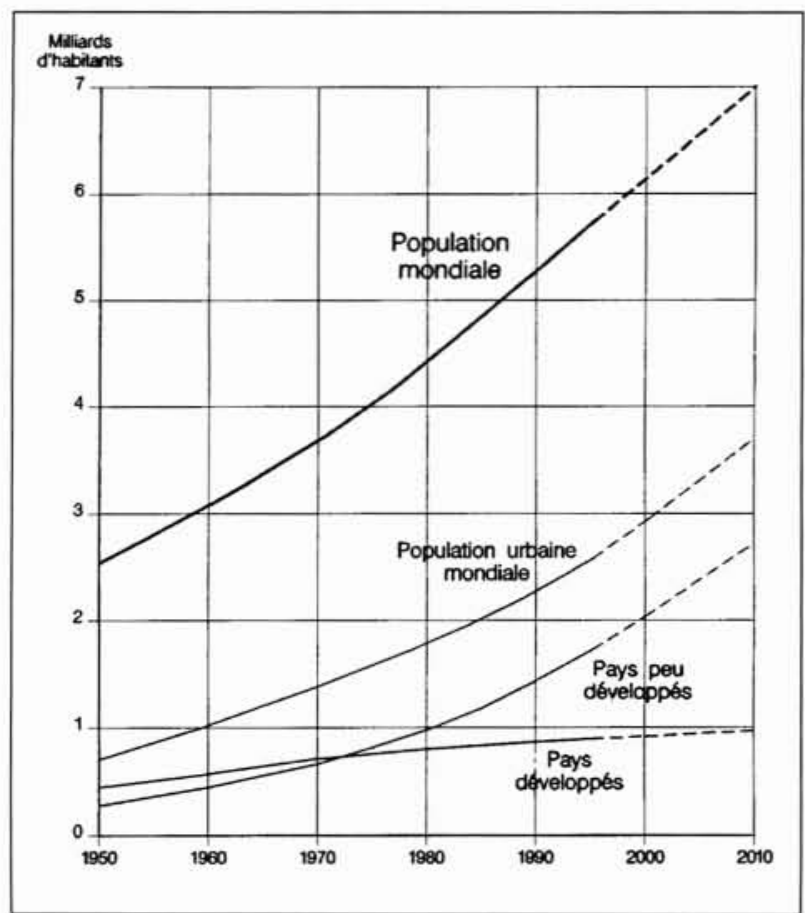

17. Evolutions et projections des populations mondiales totales et urbaines (Sources : UN, 1995).

et compte aujourd'hui près de 3 milliards d'hommes. Sa croissance a été partout plus rapide que celle des populations totales, aussi la proportion urbanisée de la population mondiale a-t-elle été elle-même croissante passant de 35 à $45 \%$ au cours des 30 dernières années et elle est en passe de dépasser $50 \%$. La population des villes est largement majoritaire $(70$ à $75 \%)$ aujourd'hui en Europe, Amérique du Nord et du Sud, Océanie. Mais, alors que cette croissance tend à se ralentir dans les pays développés, elle s'accélère au contraire dans les pays en développement dont la population urbaine a dépassé celle des pays développés après 1970 : près de 1400 millions d'êtres humains se pressent aujourd'hui dans les villes du tiers-monde, contre 880 dans celles des pays développés (Fig. 17).

C'est en Afrique et en Amérique du Sud tropicale que cette croissance a été la plus forte : les populations urbaines s'y sont multipliées par 6 entre 1950 et 1990.

Les villes ont grandi en nombre et surtout en taille. De 78 en 1950 , les villes de plus d'un million d'habitants sont devenues 222 en 1980, dont 35 de plus de 4 millions d'habitants ; elles seront 320 en 2000 et 600 en 2025 dont une cinquantaine dépasseront 10 millions de citadins. Le foisonnement de mégapoles de moins en moins contrôlables et desservies caractérise l'urbanisation du tiers-monde.

Cette urbanisation croissante a largement amplifié les demandes en eau potable, mais assez inégalement suivant les régions du monde en raison de la variété des modes et des taux de desserte et des différences de demande par tête qui leur sont en partie liées. A cet égard, un grand écart sépare les pays développés de ceux du tiers-monde.

Dans les premiers, les taux de desserte des agglomérations ont beaucoup progressé et approchent aujourd'hui $100 \%$ tandis que les demandes unitaires ont été également croissantes, tout en demeurant aujourd'hui assez variées (de 100 à $5001 /$ jour par tête) et tendent à plafonner.

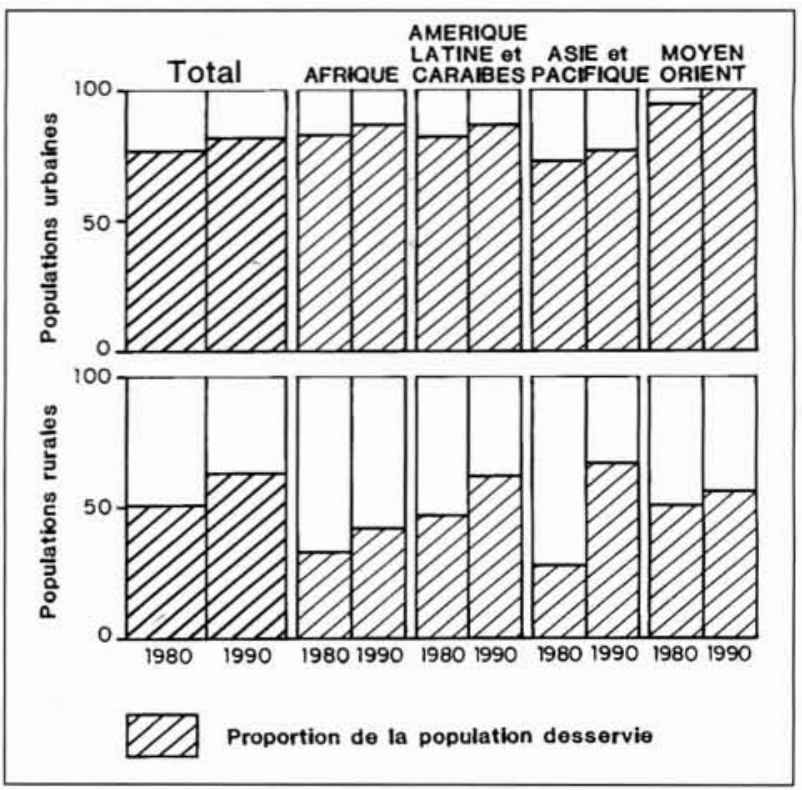

18. Variation des populations urbaines et rurales des pays en développement desservies en eau potable entre 1980 et 1990 (Source : WHO-OMS, 1991).

Dans les seconds par contre, la progression de la distribution d'eau potable par des réseaux publics ne suit pas la croissance des populations agglomérées. Les taux de desserte estimés sur des bases similaires à ceux calculés dans les pays développés ont peu progressé et ont souvent régressé.

Dans bien des villes, une faible partie seulement des habitants est branchée sur un réseau de distribution public. C'est par le biais d'une conception élargie de la desserte (proximité de bornes-fontaines, développement d'un secteur commercial de portage d'eau...) que les statistiques sur les populations "ayant accès à l'eau saine" donnent une vision plus optimiste (Fig. 18). Mais focaliser l'analyse sur les nombres d'habitants ainsi "desservis" ne donne pas la mesure des quantités d'eau distribuées ou acquises qui restent faibles par personne (ces quantités d'eau par habitant sont parfois même en régression).

Au cours de la Décennie internationale de l'eau potable promue par l'OMS (1981-1990), les populations urbaines du tiers-monde ayant accès à l'eau potable se sont effectivement accrues (de $50 \%$ ), mais celles sans accès ont augmenté aussi, dans une mesure moindre il est vrai : elles s'élèveraient encore en 1990 à 244 millions d'habitants.

Ainsi, contrairement au cas de l'irrigation, dans le secteur de l'alimentation en eau potable des populations, c'est l'urbanisation des pays développés - pourtant moins croissante que celle du tiers-monde - qui a le plus fait grossir les demandes en eau et les quantités mobilisées à cette fin.

\subsubsection{L'industrialisation}

Le développement industriel - y compris celui du secteur énergétique - est sans conteste un facteur primordial de la croissance des demandes en eau mondiales au $\mathrm{XX}^{\mathrm{e}}$ siècle, puisque les quantités utilisées par les industries dans leur ensemble auraient été multipliées par 25 depuis 1900 et par 5 depuis 1950 (Fig. 11). Elles auraient dépassé aujourd'hui le volume des demandes en eau d'irrigation du début du siècle. 


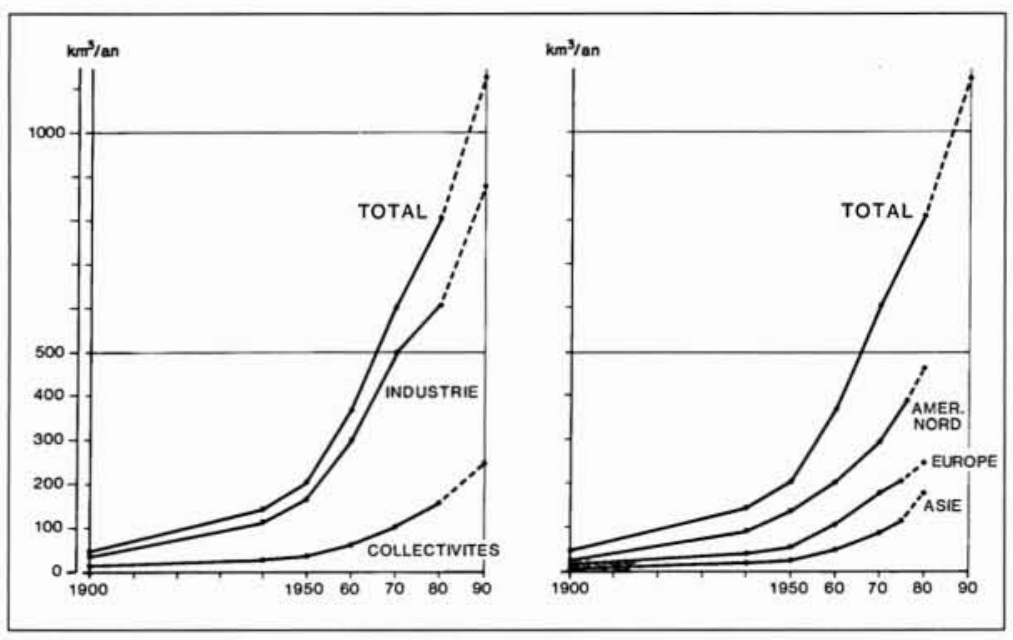

19. Evolution probable, au cours du $\mathrm{XX}$ siècle, des rejets mondiaux d'eaux résiduaires industrielles et d'eaux usées urbaines, calculés par différence entre les prélèvements et les consommations par les usages. (Sources : UNESCO, 1990 pour les secteurs et 1979 pour les continents).

Cependant, les effets de l'industrialisation sur cette croissance sont beaucoup plus disparates que ceux des autres secteurs et se prêtent beaucoup moins à une analyse globale du fait :

- d'une répartition très inégale dans le monde, accentuée par la diversité des structures, des parts prises par les différentes branches :

- des parts variées des industries desservies (et intégrées dans les facteurs de demande urbaine) ou auto-approvisionnées ;

- des progrès inégaux du recyclage suivant les pays et les branches :

- des fortes incidences des changements technologiques sur les demandes en eau (par exemple : les modes de refroidissement des centrales thermiques - Cf. encadré 2), qui ont entraîné des décroissances de nombreuses demandes unitaires (par tonne de produit) ;

- de la difficulté de relier les demandes en eau industrielles à des indicateurs macro-économiques simples (indice de production en valeur ou en volume, emplois...) ;

- de la coexistence de tendances actuelles contraires : décroissance des demandes en eau, dans les pays développés industrialisés (exemples : en France, aux États-Unis), croissance dans les pays peu développés, mais à partir de niveaux très bas.

Plus que celles de tout autre secteur, les demandes industrielles se prêtent mal à des projections par extrapolation de tendance..

\section{- 3.7 Les consommations en qualité se sont, elles aussi, accrues}

Deux facteurs ont joué en sens inverses sur l'évolution des consommations en qualité :

(1) - La croissance des rejets d'eaux usées urbaines et industrielles et des charges de matières correspondantes. Si l'on assimile ces rejets à la part des volumes d'eau prélevés non consommés par les usages pour ces deux secteurs (ce qui les surestime un peu, en y incorporant les pertes de transport avant usage), en se basant sur les essais de reconstitution déjà cités, de source UNESCO (Fig. 11), on en déduit que les flux d'eau usée urbaine et industrielle du monde entier auraient été multipliés par 20 depuis le début du siècle passant de moins de $50 \mathrm{~km}^{3} / \mathrm{an}$ en 1900 à plus de 1000 à l'heure actuelle. Contrairement aux consommations en quantité, c'est en Amérique du Nord et en Europe que ces croissances ont été les plus fortes (Fig. 19).
(2) - Les progrès des équipements et des opérations d'assainissement et d'épuration visant à neutraliser les impacts des retours d'eau, complétés parfois par des aménagements régulateurs (soutien d'étiage). Ces derniers ont été rarement à la hauteur de la croissance des utilisations et des usures de l'eau : des dégradations de qualité des eaux s'en sont suivies dans beaucoup de régions du monde.

\section{DEUX CONCLUSIONS}

- Les ressources en eau très inégalement distribuées dans le monde sont aussi inégalement utilisées. Les utilisations varient beaucoup par les objectifs et les usages, en quantité par habitant comme en proportion des ressources. Leur croissance au $\mathrm{XX}^{\mathrm{e}}$ siècle a été forte mais inégale suivant les pays et déterminée par des facteurs variés. C'est seulement depuis peu que la rareté des ressources commence à être un facteur limitant des utilisations dans une partie du monde. Aussi serait-il imprudent d'extrapoler les tendances contemporaines perceptibles pour projeter les utilisations à venir.

- L'utilisation de l'eau ne peut dissocier l'usage et l'usure. Toute demande en eau à prélever se double d'une seconde demande à la nature : reprendre et régénérer l'eau retournée après usage. L'eau sert avant tout en circulant, dans un corps humain, comme dans une ville, une usine ou un champ irrigué et drainé.

L'analyse et la prospective des besoins en eau et des utilisations ne doivent donc pas séparer, du point de vue des effets sur le milieu et par conséquent sur la ressource, les prélèvements, les consommations nettes et les retours d'eau. De même, dans la sphère économique, face aux services attendus des usages de l'eau, les charges de production et d'approvisionnement en eau ne doivent pas être dissociées de celles de l'assainissement et du traitement des eaux usées.

\section{BIBLIOGRAPHIE}

[1] DA CUNHA L.V. (1989) - Sustainable development of water resources (Inter. Sympos, on integrated approaches to water pollution problems. Lisbon, june, 34 p.).

[2] ERHARD-CASSEGRAIN A., MARGAT J. (1982) - Introduction à l'économie générale de l'eau. (Paris, Masson, 361 p.)

[3] FALKENMARK M. (1986) - Macro-Scale Water Supply / Demand Comparison on the Global Scene, (Beiträge Zur Hydrologie. Stockholm. Sonder 6, pp. 15-40). 
[4] FALKENMARK M. (1986) - Fresh water - Time for a modified approach. (AMBIO, vol. 15, $\mathrm{n}^{\circ} 4$, pp. 192-200, Stockholm)

[5] FALKENMARK M. (1988) - Implications of water scarcity for societal decision making (Proceed. intern. workshop "Water awareness in societal planning and decision making", Skokloster, Sweden, 27 June - I July 1988, I. Johansson ed. Byggforskningsradet, Swed. Council for Building Research, Stockholm, pp. 11-135).

[6] FAO (1995) - L'irrigation en Afrique en chiffres. (FAO, Water Reports/Rapports sur l'eau, 7, 336 p., Roma).

[7] FAO (1997) - Irrigation in the Near East Region in Figures. (Fao, Water reports 9, 281 p., Roma).

[8] LAUGERI L. / O.M.S. (1988) - Towards the targets : an overview of progress in the first five years of the international Drinking water supply and Sanitation Decade. (Coll. "Coût et prix de 1'eau en ville. Alimentation et assainissement". Éc. Nat. Ponts \& chaussées. Paris, déc., Presses E.N.P.C., pp. 554-562).

[9] MARGAT J. (1990) - L'économie de l'eau dans le monde : ressources, besoins, problèmes. A chacun ses problèmes d'eau. (in "Le grand livre de l'eau". Paris, Éd. La Manufacture / Cité des Sciences et de l'industrie, pp. 367-381)

[10] MARGAT J. (1992) - Répartition des ressources et des demandes en eau dans le monde. Disparités présentes et futures. (Soc. hydrot. Fr., XXIle Journées de l'Hydraulique, "L'avenir de l'eau", Paris, 15-17 sept. 1992, 16 p., Publ. La Houille Blanche 4-1993, pp. 215-224, Paris et Rev. ingénieurs Écoles nat. sup. Mines, Spécial L’Eau, nov. 1992).

[11] MARGAT J. (1992) - L'économie de l'eau dans le monde. (Con. écon. et soc., Acad. Sc/CADAS, Fr., C.R. Journée sur "Les aménagements hydrologiques, Paris, 26.05.1994, pp. 22-42, publ. Fév. 1995)

[12] MARGAT J. (1994) - Les utilisations d'eau dans le monde : État présent et essai de prospective. (Contribution au projet M-13 du PHIIV/UNESCO, Paris, 87 p.).

[13] MARGAT J. (1996) - L'alimentation en eau de l'humanité, situation et tendances présente. Prospective. (Colloque : L'eau et la vie des hommes au XXIe siècle, 26-27 mars, Paris. Les cahiers du MURS, $\mathrm{n}^{\circ} 32$, ler trim. 1997. pp. 13-33, Paris).

[14] OSS/Plan Bleu (1996) - Les indicateurs d'économie de l'eau : ressources et utilisations (Observatoire du Sahara et du Sahel/Plan Bleu, mai, 60 p. Paris, Sophia Antipolis).

[15] POSTEL S. (1984) - Water : Rethinking Management in an Age of Scarcity. (New-York, Worldwatch Paper 62).
[16] POSTEL S. (1989) - Water for Agriculture : Facing the Limits. (NewYork, Dec., Worldwatch Paper 93, 54 p.).

[17] POSTEL S. (1992) - Last Oasis. Facing water scarcity. (New-York, London, Worldwatch Institute, Norton \& Co., 240 p.).

[18] SHIKLOMANOV I.A. (1990) - Assessment of Water Ressources and Water Available in the World. (State hydrological Institute of Russia/UNESCO, February, 127 p.).

[19] TENIERE - BUCHOT P.F. (1992) - L'avenir de l'eau : évolution des besoins et des usages. (Soc. hydrot. Fr., XXlle journées de l'Hydraulique, "L'avenir de l'eau". Paris, $15-17$ sept. 1992, Publ. La Houille Blanche 4-1993, pp. 225-232).

[20] COLLECTIF, KORZUN V.I. ed. $(1974,1978)$ - World Water Balance and Water Resources of the Earth (Com. URSS/PHI. 1974. UNESCO, 1978, Paris).

[21] COLLECTIF/FAO. (1988) - Irrigation Practice and Water Management (Rome, Irrigation and Drainage Paper I, Doneen \& Westcot).

[22] ANONYME/FAO. (1990) - An International Action Programme on Water and Sustainable Agricultural Development. (Rome, FAO,, 42 p.)

[23] ANONYME/WHO/OMS (1991) - The international drinking water supply and sanitation decade : review of decade progress (as at December 1990). (WHO Division of Environnemental Health, Geneva).

[24] ANONYME/UN-ECE (1993) - The Environnement in Europe and North-America : Annotated statistics 1992 (UN, Economic Comission for Europe, New-York, Genève).

[25] ANONYME/UN (1995) - World Urbanization Prospects : The 1994 Revisions. Estimates and Projections of Urban and Rural Populations. and of Urban Agglomerations. (UN, Dep for Economic and Social information and Policy Analysis. Population Division, ST/Ser.A/150. New-York).

[26] ANONYME/WORLD RESOURCES INSTITUTE (1996) - World Resources 1996-1997, Part III, 11, Freshwater \& Table 213.1 (Washing. ton, World Resources Institute / Inter. Inst. for Environment and Development).

[27] ANONYME (1996) - Les prélèvements et consommations d'eau en France de 1981 à 1990 (par bassins, usages et origines). (Neuillys/Seine, juillet, Minist. Environ. / Dir. Eau, 62 p.)

[28] ANONYME/OCDE (1997) - Données OCDE sur l'environnement Compendium 1997 (OCDE, Paris). 